\title{
Practical Aspects of Solving Hybrid Bayesian Networks Containing Deterministic Conditionals*
}

\author{
Prakash P. Shenoy ${ }^{1 \dagger}$, Rafael Rumí ${ }^{\ddagger} ;$ Antonio Salmerón ${ }^{2 \S}$ \\ ${ }^{1}$ School of Business, University of Kansas, \\ Lawrence, KS 66045-7601 USA \\ ${ }^{2}$ Department of Mathematics, University of Almería, \\ E-04120 Almería, Spain
}

\begin{abstract}
In this paper we discuss some practical issues that arise in solving hybrid Bayesian networks that include deterministic conditionals for continuous variables. We show how exact inference can become intractable even for small networks, due to the difficulty in handling deterministic conditionals (for continuous variables). We propose some strategies for carrying out the inference task using mixtures of polynomials and mixtures of truncated exponentials. Mixtures of polynomials can be defined on hypercubes or hyper-rhombuses. We compare these two methods. A key strategy is to re-approximate large potentials with potentials consisting of fewer pieces and lower degrees/number of terms. We discuss several methods for re-approximating potentials. We illustrate our methods in a practical application consisting of solving a stochastic PERT network.
\end{abstract}

\section{Introduction}

Hybrid Bayesian networks are Bayesian networks (BNs) that include a mix of discrete and continuous random variables. A random variable is discrete if its state space is countable, and is continuous otherwise. In a BN, each variable is associated with a conditional distribution for it given each state of its parents. A conditional distribution for a variable is said to be deterministic if its variances are all zeroes (for each state of its parents).

The first proposal of an exact algorithm for hybrid BNs was developed for the case in which the joint distribution of all variables is a mixture of Gaussians (MoG). ${ }^{1}$ Some limitations of the MoG model are that it is restricted to

\footnotetext{
*Preliminary versions of this paper were presented at the ISDA'2011 conference and PGM'2012 workshop

${ }^{\dagger}$ Author to whom all correspondence should be addressed; e-mail:pshenoy@ku.edu

${ }^{\ddagger}$ rrumi@ual.es

$\S$ antonio.salmeron@ual.es
} 
network topologies in which discrete variables do not have continuous parents, and each conditional for a continuous variable has to be a conditional linear Gaussian, i.e., a Gaussian distribution whose mean is a linear function of its continuous parents, and whose variance is a constant. Also, during the solution phase, continuous variables have to be marginalized before discrete ones and this restriction can lead to large cliques, ${ }^{2}$ and consequently large memory storage requirements. It has been shown that inference in MoG networks is NP-hard, even for network structures for which inference in the discrete case is easy. ${ }^{2}$

A more general technique is based on the use of mixtures of truncated exponentials (MTEs). ${ }^{3}$ The MTE model does not impose any topological restrictions on its networks, and is compatible with any efficient algorithm for exact inference that requires only the combination and marginalization operations, such as the Shenoy-Shafer ${ }^{4,5}$ and variable elimination methods. ${ }^{6}$ Furthermore, MTEs have shown a remarkable ability for fitting many commonly used univariate probability density functions (PDFs). ${ }^{7}$

Hybrid BNs can also be solved by discretizing the continuous variables, so that all the existing methodology for discrete BNs can be applied with any further modification. The most prominent proposal in this direction is the so-called dynamic discretization, ${ }^{8}$ where inference is carried out iteratively in an any-time manner, seeking for better representations of high density areas. A study of the complexity of the MTE approach versus discretization can be found in existing literature. ${ }^{9,10}$

The most recent proposal for dealing with hybrid BNs is based on the use of mixtures of polynomials (MOPs). ${ }^{11}$ Like MTEs, MOPs have high expressive power, but the latter are superior in dealing with deterministic conditionals for continuous variables. ${ }^{5,11}$ Also, a MOP approximation of a PDF can be easily found using Lagrange interpolating polynomials with Chebyshev points. ${ }^{12}$ Both MTEs and MOPs can be seen as instantiations of a more general framework known as mixtures of truncated basis functions (MoTBFs). ${ }^{13}$

In this paper, we discuss some practical issues that have to be addressed in order to make inference in hybrid BNs tractable, even for small problems, when deterministic conditionals are present. Dynamic discretization can also be used in this context, ${ }^{8,14}$ but the approach we follow here is based on the use of MOPs and MTEs as discretization is in fact a particular case of these models. ${ }^{13}$ A key strategy is re-approximation of MOPs (MTEs) with fewer pieces and lower degrees (fewer terms) during the solution phase. We compare the sizes, computation time and accuracy of MOPs defined on hypercubes and hyper-rhombuses. We compare the performance of MOPs and MTEs in this context, through an example arising from a stochastic PERT network. ${ }^{15}$ We also carry out an experiment illustrating how the complexity grows with the size of the model. 
Contributions The main contributions of this paper are as follows. First, in the case of MOPs, they can be defined on either hypercubes or hyperrhombuses when deterministic conditionals are present in the network. In this paper, we do a small comparison of these two possibilities. Second, in the case of MOPs or MTEs, we describe a re-approximation method by dropping pieces. Third, in the case of MOPs, we describe a re-approximation method using Lagrange interpolating polynomials with Chebyshev points. ${ }^{12}$ Fourth, in the case of MTEs, we describe a re-approximation method using numeric least squares. Fifth, we demonstrate the efficacy of the reapproximation methods by solving a small hybrid Bayesian network with 2 discrete variables, and 10 continuous variables of which 4 have deterministic conditionals. We were unable to solve this problem without using re-approximations.

Limitations Some limitations of our work are as follows. First, we do not describe the worst-case complexity of inference in hybrid Bayesian networks with deterministic conditionals using MOPs/MTEs. Instead, we carry out an experiment illustrating how the complexity grows with the size of the model. Due to the fact that MOP/MTE-based methods are more general than MoG methods, we suspect that it is at least as bad as MoG networks, if not worse. Second, although we do a small comparison of hypercubes and hyper-rhombuses in the case of MOPs, a more systematic study is needed with more examples. Based on our limited set of experiments, we believe that hypercubes are more tractable than hyper-rhombuses, as long as an appropriate approximation can be found for each hypercube. Third, for all the re-approximations methods we have described, there are many judgments that have to be made for which we have no systematic rules for doing so. Fourth, it would be useful to know approximately the size of problems that can be solved using the re-approximation methods. Fifth, it would be useful to have some error bounds on the results of inference using MOP/MTEmethods, with or without re-approximations. All of these limitations need further research.

\section{MTEs and MOPs}

We will use uppercase letters to denote random variables, and boldfaced uppercase letters to denote random vectors, e.g. $\mathbf{X}=\left\{X_{1}, \ldots, X_{n}\right\}$, and its state space will be written as $\Omega_{\mathbf{X}}$. Lowercase letters $x$ (or $\mathbf{x}$ ) will denote elements of $\Omega_{X}$ (or $\Omega_{\mathbf{X}}$ ). The MTE model ${ }^{3}$ is defined as follows.

Definition 1. Let $\mathbf{X}$ be a mixed $n$-dimensional random vector. Let $\mathbf{Y}=$ $\left(Y_{1}, \ldots, Y_{d}\right)^{\top}$ and $\mathbf{Z}=\left(Z_{1}, \ldots, Z_{c}\right)^{\top}$ be its discrete and continuous parts respectively. A function $f: \Omega_{\mathbf{X}} \mapsto \mathbb{R}_{0}^{+}$is a mixture of truncated exponentials 
(MTE) potential if for each fixed value $\mathbf{y} \in \Omega_{\mathbf{Y}}$ of the discrete variables $\mathbf{Y}$, the potential over the continuous variables $\mathbf{Z}$ is defined as:

$$
f(\mathbf{z})=a_{0}+\sum_{i=1}^{m} a_{i} \exp \left\{\mathbf{b}_{i}^{\top} \mathbf{z}\right\},
$$

for all $\mathbf{z} \in \Omega_{\mathbf{Z}}$, where $a_{i} \in \mathbb{R}$ and $\mathbf{b}_{i} \in \mathbb{R}^{c}, i=1, \ldots, m$. We also say that $f$ is an MTE potential if there is a partition $D_{1}, \ldots, D_{k}$ of $\Omega_{\mathbf{Z}}$ into hypercubes and in each one of them, $f$ is defined as in Eq. (1). In this case, we say $f$ is a k-piece, $m$-term MTE potential.

Mixtures of polynomials (MOPs) were initially proposed as modeling tools for hybrid BNs. ${ }^{11}$ The original definition is similar to MTEs, in the sense that they are piecewise functions defined on hypercubes. A more general definition, where the hypercube condition is relaxed, can be stated as follows. ${ }^{12}$

Definition 2. Let $\mathbf{X}, \mathbf{Y}$ and $\mathbf{Z}$ be as in Definition 1. A function $f: \Omega \mathbf{X} \mapsto$ $\mathbb{R}_{0}^{+}$is a mixture of polynomials (MOP) potential if for each fixed value $\mathbf{y} \in \Omega_{\mathbf{Y}}$ of the discrete variables $\mathbf{Y}$, the potential over $\mathbf{Z}$ is defined as:

$$
f(\mathbf{z})=P(\mathbf{z}),
$$

for all $\mathbf{z} \in \Omega_{\mathbf{Z}}$, where $P(\mathbf{z})$ is a multivariate polynomial in variables $\mathbf{Z}=$ $\left(Z_{1}, \ldots, Z_{c}\right)^{\top}$. We also say that $f$ is a MOP potential if there is a partition $D_{1}, \ldots, D_{k}$ of $\Omega_{\mathbf{Z}}$ into hyper-rhombuses and in each one of them, $f$ is defined as in Eq. (2).

The fact that the elements in the partition are hyper-rhombuses, means that for any ordering of the variables $Z_{1}, \ldots, Z_{c}$, for each $D_{i}$ it holds that $l_{1 i} \leq z_{1} \leq u_{1 i}, l_{2 i}\left(z_{1}\right) \leq z_{2} \leq u_{2 i}\left(z_{1}\right), \ldots, l_{c i}\left(z_{1}, \ldots, z_{c-1}\right) \leq z_{c} \leq u_{c i}\left(z_{1}, \ldots, z_{c-1}\right)$, where $l_{1 i}$ and $u_{1 i}$ are constants, and $l_{j i}\left(z_{1}, \ldots, z_{j-1}\right)$ and $u_{j i}\left(z_{1}, \ldots, z_{j-1}\right)$ are linear functions of $z_{1}, \ldots, z_{j-1}$ for $j=2, \ldots, c$, and $i=1, \ldots, k$. Figure 1 shows the difference between a hypercube and a hyper-rhombus.

MTEs and MOPs are closed under multiplication, addition, and integration. However, integrating over hyper-rhombuses is in general more complex than over hypercubes. The advantage is that by using hyper-rhombuses, it is easier to represent models such as the conditional linear Gaussian, where the conditional distribution of a variable may depend on the values of its continuous parents in the network. Unfortunately, MTEs cannot be defined on hyper-rhombuses, as the integration operation would not remain closed for that class. 

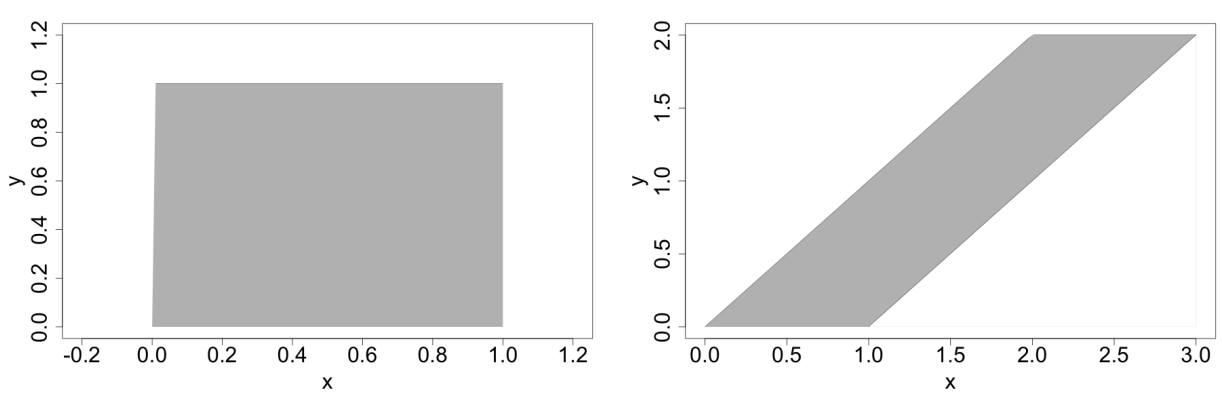

Figure 1: A hypercube defined by $0 \leq x \leq 1,0 \leq y \leq 1$ (left) and a hyperrhombus defined by $0 \leq y \leq 2, y \leq x \leq y+1$ (right). Note that the borders of the region are not constant in the hyper-rhombus case.

\section{Inference in Hybrid BNs with Deterministic Con- ditionals}

The inference task in hybrid BNs with deterministic conditionals has been already studied. ${ }^{5}$ Traditional algorithms for inference in hybrid BNs rely on the assumption that all the conditionals in the network belong to the same class, and that the operations used during inference are closed within the same class. This is not necessarily the case for deterministic conditionals.

For instance, assume we have a $\mathrm{BN}$ with three continuous variables $B, C$ and $D$, such that $D=\max \{B, C\}$. In such case, existing procedures ${ }^{5}$ cannot be directly applied. However, the max deterministic function can be converted to a linear function by introducing an auxiliary variable $A$ with two states $a$ and $n a$, which denote whether $B \geq C$ or $B<C$, respectively, obtaining the equivalent representation displayed in the right hand side of Figure 2, where $D=B$ if $A=a$ and $D=C$ if $A=n a$. Note that after the transformation, the distribution is the same as in the original network. However, the price to pay is the introduction of additional complexity, as there will be a new variable with as many parents as de deterministic variable. This gives an idea of the increased complexity when dealing with deterministic conditionals with respect to plain hybrid BNs.

Whether or not a deterministic conditional can be properly handled, depends on the model used. We will analyze the situation from the point of view of MTEs and MOPs.

\subsection{MTE Representation of Conditionals}

MTEs can be used to accurately approximate several univariate distributions. ${ }^{7}$ The approximation of conditional densities using MTEs is more dif- 

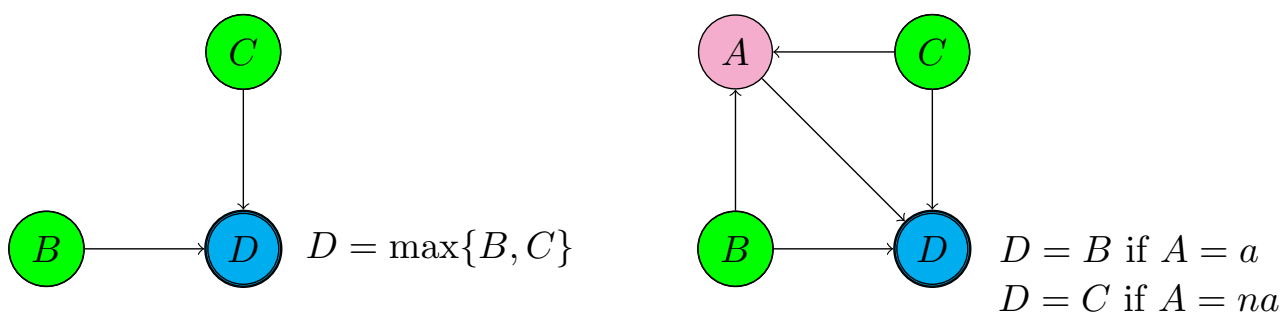

Figure 2: A $\max$ conditional (left) and its transformation (right).

ficult, ${ }^{16}$ as the hypercube condition in the definition of an MTE function means that the value of a continuous parent has to be a constant. ${ }^{17}$ Therefore, this implies that a conditional density is approximated by MTEs by partitioning the state space of the continuous parents into hypercubes, and then fitting a univariate MTE in each hypercube. The resulting conditional MTE density is called a mixed tree. ${ }^{18}$

An additional problem is found when attempting to deal with deterministic conditionals. MTEs are not closed with respect to the convolution operation required by the sum conditional. Consider two independent random variables $X_{1}, X_{2} \sim \operatorname{Exp}(\mu=1)$ and $Y=X_{1}+X_{2}$. The marginal PDF of $Y$ is $\operatorname{Gamma}[r=2, \mu=1]$, which is not an MTE function. The reason is that such a marginal is obtained through the so-called convolution operation as

$$
f_{Y}(y)=\int_{-\infty}^{\infty} f_{X_{1}}\left(x_{1}\right) f_{X_{2}}\left(y-x_{1}\right) \mathrm{d} x_{1}=\int_{0}^{y} e^{-x_{1}} e^{-\left(y-x_{1}\right)} \mathrm{d} x_{1}=y e^{-y}
$$

for $y>0$, which is not an MTE according to Definition 1. This is a consequence of the fact that even though $f_{X_{2}}\left(x_{2}\right)$ is defined on hypercubes, $f_{X_{2}}\left(y-x_{1}\right)$ is no longer defined on hypercubes, and therefore, the limits of integration in Eq. (3) are not all constants.

One solution to this problem is to approximate the function $f_{X_{2}}\left(y-x_{1}\right)$ on hypercubes using a mixed tree approach. ${ }^{18}$ In order to illustrate the mixed tree approach, consider a hybrid BN formed by variables $X_{1}, X_{2}$ and $X_{3} . X_{1} \sim N(3,1), X_{2} \mid x_{1} \sim N\left(6+2 x_{1}, 2^{2}\right)$ and $X_{3}=X_{1}+X_{2}$. If $Z \sim$ $N(0,1), f(\cdot)$ is an MTE approximation of the PDF of $Z$, and $Y=\sigma Z+\mu$, where $\sigma>0$ and $\mu$ are real constants, then $Y \sim N\left(\mu, \sigma^{2}\right)$, and an MTE approximation of the PDF of $Y$ is given by $g(y)=\frac{1}{|\sigma|} f\left(\frac{y-\mu}{\sigma}\right)$. Suppose $f(\cdot)$ is a 2-piece MTE approximation of the PDF of $N(0,1)$ on the domain $(-3,3)$, where the two pieces are $(-3,0)$ and $[0,3) .{ }^{7}$ Then, $g_{1}\left(x_{1}\right)=f\left(x_{1}-3\right)$ is an MTE approximation of the PDF of $X_{1} \sim N(3,1)$ on the domain $(0,6)$. However, $g_{2}\left(x_{1}, x_{2}\right)=f\left(\frac{x_{2}-6-2 x_{1}}{2}\right) / 2$ is not an MTE since it would be defined on regions such as $-3<\frac{x_{2}^{2}-6-2 x_{1}}{2}<0$, which are not hypercubes. So we partition the domain of $X_{1}$ into equal-size intervals, and assume that 
$x_{1}$ is a constant in each interval equal to the mid-point of the interval. Thus, $g_{2 c}\left(x_{1}, x_{2}\right)$, as described in Eq. (4), is a 3-point mixed tree MTE approximation of $g_{2}\left(x_{1}, x_{2}\right)$ representing the conditional PDF of $X_{2}$ given $x_{1}$.

$$
g_{2 c}\left(x_{1}, x_{2}\right)= \begin{cases}f\left(\frac{x_{2}-8}{2}\right) / 2 & \text { if } 0<x_{1}<2, \\ f\left(\frac{x_{2}-12}{2}\right) / 2 & \text { if } 2 \leq x_{1}<4, \\ f\left(\frac{x_{2}-16}{2}\right) / 2 & \text { if } 4 \leq x_{1}<6 .\end{cases}
$$

Notice that this mixed-tree method can also be used with MOPs. Next, we wish to compute the marginal PDF of $X_{3}$. The conditional associated with $X_{3}$ is $g_{3}\left(x_{1}, x_{2}, x_{3}\right)=\delta\left(x_{3}-x_{1}-x_{2}\right)$, where $\delta$ is the Dirac delta function ( see $^{5}$ for a detailed description on the use of Dirac delta functions for handling deterministic relationships). To compute the marginal of $X_{3}$, we first marginalize $X_{2}$ and then $X_{1}$. The result after marginalizing $X_{2}$ is $\left.g_{4}\left(x_{1}, x_{3}\right)=\int_{-\infty}^{\infty} g_{2 c}\left(x_{1}, x_{2}\right) \delta\left(x_{3}-x_{1}-x_{2}\right)\right) \mathrm{d} x_{2}=g_{2 c}\left(x_{1}, x_{3}-x_{1}\right)$, which represents the conditional of $X_{3}$ given $x_{1}$.

Now we notice that $g_{4}\left(x_{1}, x_{3}\right)$ is not defined on hypercubes anymore since we have regions such as $a \leq x_{3}-x_{1}<b$, where $a$ and $b$ are constants. So we approximate $g_{4}\left(x_{1}, x_{3}\right)$ by $g_{5 c}\left(x_{1}, x_{3}\right)$ using mixed trees as follows:

$$
g_{5 c}\left(x_{1}, x_{3}\right)= \begin{cases}g_{2 c}\left(1, x_{3}-1\right) & \text { if } 0<x_{1}<2, \\ g_{2 c}\left(3, x_{3}-3\right) & \text { if } 2 \leq x_{1}<4, \\ g_{2 c}\left(5, x_{3}-5\right) & \text { if } 4 \leq x_{1}<6 .\end{cases}
$$

Notice that $g_{5 c}\left(x_{1}, x_{3}\right)$ is an MTE function since it is defined on hypercubes. Next we marginalize $X_{1}$ as follows (resulting in the marginal PDF $g_{6 c}(\cdot)$ of $\left.X_{3}\right)$ :

$$
g_{6 c}\left(x_{3}\right)=\int_{-\infty}^{\infty} g_{1}\left(x_{1}\right) g_{5 c}\left(x_{1}, x_{3}\right) \mathrm{d} x_{1} .
$$

Since MTEs are closed under multiplication and integration, $g_{6 c}(\cdot)$ is an MTE function. A cost of the mixed-tree approach to maintain the hypercube nature of the pieces is the increase in the number of pieces. Thus, if $f(\cdot)$ is a 1-piece MTE approximation of the PDF of $N(0,1)$, then $g_{1}\left(d_{1}\right)$ is a 1-piece MTE potential, $g_{2 c}\left(d_{1}, d_{3}\right)$ is a 3 -piece MTE potential and $g_{5 c}\left(d_{1}, c_{3}\right)$ is a 3 -piece MTE potential. Another cost is loss of accuracy. We have used 3point mixed trees for our illustration. We could use more points to improve accuracy of the computed potentials, but this would mean more pieces. We will discuss this in more detail in Section 3.3 for the case of MOPs.

\subsection{MOP Representation of Conditionals}

The problem of fitting univariate and multi-variate PDFs using MOPs has already been approached using the Taylor series approximation of differen- 
tiable functions. ${ }^{11}$ More recently, the ability of MOPs to fit multivariate conditional linear Gaussian distributions was significantly improved by means of allowing the functions to be defined into hyper-rhombuses rather than into hypercubes. ${ }^{12}$ Also, an improved method for finding MOP approximations based on Lagrange interpolating polynomials with Chebyshev points has been proposed. ${ }^{12}$

To illustrate this improvement, consider the BN used in Section 3.1 formed by three continuous variables $X_{1}, X_{2}$ and $X_{3}$. Suppose $f(\cdot)$ is a 2-piece, 3-degree MOP approximation of the PDF of $N(0,1)$ on the domain $(-3,3) .{ }^{12}$ Then, $g_{1}\left(x_{1}\right)=f\left(x_{1}-3\right)$ is a 2-piece, 3-degree MOP approximation of the PDF of $X_{1} \sim N(3,1)$ on the domain $(0,6)$. Finally, $g_{2}\left(x_{1}, x_{2}\right)=f\left(\frac{x_{2}-6-2 x_{1}}{2}\right) / 2$ is a 2-piece, 3-degree MOP approximation of the conditional PDF of $X_{2}$ given $x_{1}$. Notice that $g_{2}\left(x_{1}, x_{2}\right)$ is defined on hyper-rhombuses, e.g., $-3<\frac{x_{2}-6-2 x_{1}}{2}<0$, etc, and not on hypercubes.

Unlike MTEs, MOPs are closed under the operations required for sum conditionals. Thus, after the elimination of $X_{2}, g_{5}\left(x_{1}, x_{3}\right)=g_{2}\left(x_{1}, x_{3}-x_{1}\right)$ is a MOP, and after the elimination of $X_{1}, g_{6}\left(x_{3}\right)=\int_{-\infty}^{\infty} g_{1}\left(x_{1}\right) g_{5}\left(x_{1}, x_{3}\right) \mathrm{d} x_{1}$ is also a MOP. So in the case of MOPs, we have a choice of using MOPs defined on hypercubes (using mixed trees) or on hyper-rhombuses. In the next subsection, we compare the two alternatives in terms of the sizes of MOP potentials (pieces and degrees), computation time, and accuracy of resulting MOP potentials. All computations were done in Mathematica ${ }^{\circledR} \mathrm{v}$. 8.0.4, running on an Apple iMac with $3.4 \mathrm{GHz}$ Intel Core i7 processor and 16 GB memory. ${ }^{1}$

\subsection{Hypercube vs. Hyper-rhombus MOP Representations of Gaussian PDFs}

In order to compare the hypercube and the hyper-rhombus approximations of Gaussian PDFs, we do a small experiment. Consider again the BN formed by $X_{1}, X_{2}$ and $X_{3}$. We will represent the PDFs of $X_{1}$ and $X_{2}$ using hypercubes and hyper-rhombuses. We will compute the marginal PDFs of $X_{2}$ and $X_{3}$, and compare the sizes of resulting MOP marginals, time required for the computation of marginals, and the quality of the approximation.

\subsubsection{Using Hypercube MOPs}

We start with $f(z)$, a 2-piece, 3-degree MOP approximation of the PDF of $N(0,1)$ on the interval $(-3,3) .{ }^{11}$ Then as discussed before, $g_{1}\left(x_{1}\right)=$ $f\left(x_{1}-3\right)$ is a 2-piece, 3 -degree MOP approximation of the PDF of $N(3,1)$ on $(0,6)$, and $g_{2 c}\left(x_{1}, x_{2}\right)$ as defined in Eq. (4) is a 6-piece, 3 -degree hypercube MOP approximation of the conditional PDF of $X_{2}$ given $X_{1}$. The marginal

\footnotetext{
${ }^{1}$ The Mathematica $^{\circledR}$ notebooks used in this paper can be downloaded from http://elvira.ual.es/DetCond
} 

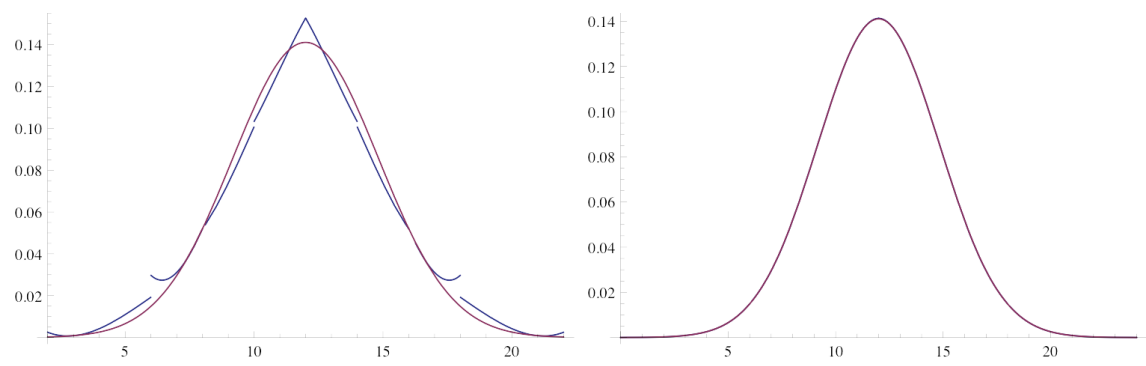

Figure 3: Left: A plot of $g_{4 c}(\cdot)$ (blue) overlaid on the PDF of $N(12,8)$ truncated to $(2,22)$ (red). Right: A plot of $g_{4}(\cdot)$ (blue) overlaid on the PDF of $N(12,8)$ truncated to $(0,24)$ (red).

PDF of $X_{2}$ is given by

$$
g_{4 c}\left(x_{2}\right)=\int_{-\infty}^{\infty} g_{1}\left(x_{1}\right) g_{2 c}\left(x_{1}, x_{2}\right) \mathrm{d} x_{1} .
$$

It takes approximately 1.41 seconds to compute the integral above and results in a 8-piece, 3-degree MOP approximation of the PDF of $X_{2}$ on the interval $(2,22)$. The exact marginal distribution of $X_{2}$ is $N(12,8)$. A plot of $g_{4 c}$ overlaid on the plot of the PDF of $N(12,8)$ truncated to $(2,22)$ is shown at the left in Figure 3.

We will measure the accuracy of a PDF with respect to another defined on the same domain by four different measures, the Kullback-Leibler (KL) divergence, maximum absolute deviation, absolute error of the mean, and absolute error of the variance. These measures are defined as follows. ${ }^{12}$

If $f$ is a PDF on the interval $(a, b)$, and $g$ is a PDF that is an approximation of $f$ such that $g(x)>0$ for $x \in(a, b)$, then the $K L$ divergence between $f$ and $g$, denoted by $K L(f, g)$, is defined as

$$
K L(f, g)=\int_{a}^{b} \ln \left(\frac{f(x)}{g(x)}\right) f(x) d x .
$$

$K L(f, g) \geq 0$, and $K L(f, g)=0$ if and only if $g(x)=f(x)$ for all $x \in(a, b)$.

The maximum absolute deviation between $f$ and $g$, denoted by $M A D(f, g)$, is given by:

$$
M A D(f, g)=\sup \{|f(x)-g(x)|: a<x<b\} .
$$



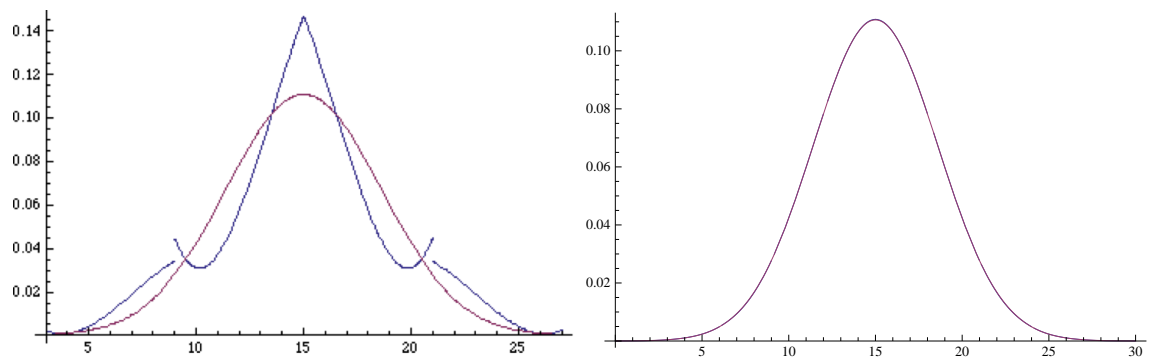

Figure 4: Left: A plot of $g_{6 c}(\cdot)$ (blue) overlaid on the PDF of $N(15,13)$ truncated to $(3,27)$ (red). Right: A plot of $g_{6}(\cdot)$ (blue) overlaid on the PDF of $N(15,13)$ truncated to $(0,30)$ (red). Note that $g_{6 c}(\cdot)$ is computed using hypercube MOPs while $g_{6}(\cdot)$ is obtained using hyper-rhombuses MOPs.

The absolute error of the mean, denoted by $A E M(f, g)$, and the absolute error of the variance, denoted by $A E V(f, g)$, are given by:

$$
A E M(f, g)=|E(f)-E(g)|, \quad A E V(f, g)=|V(f)-V(g)|,
$$

where $E(\cdot)$ and $V(\cdot)$ stand for expected value and variance of a PDF.

The goodness of fit measures for $g_{4 c}$ vs. $f_{X_{2}}$, the PDF of $N(12,8)$ truncated to $(2,22)$, are displayed in Table 3 , hypercube column.

Next, we compute the PDF of $X_{3}$. After marginalization of $X_{2}$, we have $g_{5}^{\prime}\left(x_{1}, x_{3}\right)=g_{2 c}\left(x_{1}, x_{3}-x_{1}\right)$, which is not defined on hypercubes. So we approximate $g_{5}^{\prime}$ by $g_{5 c}\left(x_{1}, x_{3}\right)$ as defined in Eq. (5). After marginalization of $X_{1}$, we get $g_{6 c}\left(x_{3}\right)$, which is a 6 -piece, 3 -degree MOP approximation of the PDF of $X_{3}$ defined on the domain $(3,27)$. It takes approximately $1.31 \mathrm{sec}-$ onds to compute $g_{6 c}(\cdot)$. The exact marginal distribution of $X_{3}$ is $N(15,13)$. A plot of $g_{6 c}$ overlaid on the plot of the PDF of $N(15,13)$ truncated to $(3,27)$ is shown at the left in Figure 4.

The goodness of fit measures for $g_{6 c}$ vs. $f_{X_{3}}$, the PDF of $N(15,13)$ truncated to [3, 27], are displayed in Table 3, hypercube column.

\subsubsection{Using Hyper-rhombus MOPs}

As in the case of hypercubes, we start with $f(\cdot)$, a 2-piece, 3-degree MOP approximation of the PDF of $N(0,1)$. Then $g_{1}\left(x_{1}\right)=f\left(x_{1}-3\right)$ is a MOP approximation of the PDF of $X_{1}$, and $g_{2}\left(x_{1}, x_{2}\right)=f\left(\frac{x_{2}-6-2 x_{1}}{2}\right) / 2$ is a hyper-rhombus MOP approximation of the conditional PDF of $X_{2} \mid x_{1}$. The marginal PDF of $X_{3}$ is computed as

$$
g_{4}\left(x_{2}\right)=\int_{-\infty}^{\infty} g_{1}\left(x_{1}\right) g_{2}\left(x_{1}, x_{2}\right) \mathrm{d} x_{1} .
$$

It takes approximately 3.44 seconds to do this integral, and $g_{4}(\cdot)$ is computed as a 4 -piece, 7 -degree MOP on the domain $(0,24)$. The exact marginal 
distribution of $X_{2}$ is $N(12,8)$. A plot of $g_{4}$ overlaid on the plot of the PDF of $N(12,8)$ truncated to $(0,24)$ is shown at the right in Figure 3.

The goodness of fit measures for $g_{4}$ vs. $f_{X_{2}}^{\prime}$, the PDF of $N(12,8)$ truncated to [0,24], are displayed in Table 3, hyper-rhombus column.

Finally, we compute the marginal PDF of $X_{3}$ as follows:

$$
g_{6}\left(x_{3}\right)=\int_{-\infty}^{\infty} g_{1}\left(x_{1}\right) g_{2}\left(x_{1}, x_{3}-x_{1}\right) \mathrm{d} x_{1} .
$$

It takes approximately 4.98 seconds to do the integration in Eq.(12), and $g_{6}(\cdot)$ is computed as a 9-piece, 7 -degree MOP on the domain $(0,30)$. The exact marginal distribution of $X_{3}$ is $N(15,13)$. A plot of $g_{6}$ overlaid on the plot of the PDF of $N(15,13)$ truncated to $(0,30)$ is shown at the right in Figure 4 . The goodness of fit measures for $g_{6}$ vs. $f_{X_{3}}^{\prime}$, the PDF of $N(15,13)$ truncated to $(0,30)$, are displayed in Table 3 , hyper-rhombus column.

\subsubsection{Comparison}

We will compare the computed marginals of $X_{2}$ and $X_{3}$ in terms of the sizes of MOPs, time required for computation, and accuracy.

Sizes of MOPs. Consider the sizes of the MOP approximations of the marginals of $X_{2}$ and $X_{3}$ displayed in Table 1 .

\begin{tabular}{l|cc} 
Size of $M O P s$ & Hypercube & Hyper-rhombus \\
\hline Marg. PDF of $X_{2}$ & 8-piece & 4-piece \\
$g_{4 c}$ vs. $g_{4}$ & 3-degree & 7-degree \\
\hline Marg. PDF of $X_{3}$ & 6-piece & 9-piece \\
$g_{6 c}$ vs. $g_{6}$ & 3-degree & 7-degree
\end{tabular}

Table 1: Sizes of the MOP approximations of the marginals of $X_{2}$ and $X_{3}$.

For the marginal PDF of $X_{2}$, the hypercube MOP $g_{4 c}(\cdot)$ has more pieces but fewer degrees than the corresponding MOP $g_{4}(\cdot)$. To see why, $g_{4 c}(\cdot)$ is computed as described in Eq. (7). $g_{1}\left(x_{1}\right)$ is a 2-piece, 3-degree MOP and $g_{2 c}\left(x_{1}, x_{2}\right)$ is a 6 -piece, 3 -degree MOP. After multiplication, $g_{1}\left(x_{1}\right) g_{2 c}\left(x_{1}, x_{2}\right)$ is a 8-piece, 6-degree MOP defined on hypercubes. After integration with respect to $d_{1}$, the degrees associated with $d_{1}$ disappear, but none of the pieces do, resulting in a 8-piece, 3 -degree MOP $g_{4 c}\left(x_{2}\right)$. On the other hand, $g_{4}(\cdot)$ is defined as in Eq.(11). $g_{1}\left(x_{1}\right)$ is a 2-piece, 3-degree MOP, and $g_{2}\left(x_{1}, x_{2}\right)$ is a 2-piece, 3-degree MOP defined on hyper-rhombus. After multiplication, $g_{1}\left(x_{1}\right) g_{2}\left(x_{1}, x_{2}\right)$ is a 4-piece, 6-degree MOP defined on hyper-rhombus regions. After integration with respect to $x_{1}$, the result is a 6-piece, 7-degree MOP. Two of the six pieces are defined on singleton points $\left(x_{2}=18\right.$, and $\left.x_{2}=22\right)$. The reason why the degree increases to 7 is because when integrating a MOP defined on a rhombus, the limits of integration are in terms 
of $x_{2}$, and since $\int x_{1}^{n} \mathrm{~d} x_{1}=\frac{x_{1}^{n+1}}{n+1}$, the degree of the resulting MOP increases from 6 to 7 .

For the marginal PDF of $X_{3}$, the hypercube MOP $g_{6 c}(\cdot)$ has fewer pieces and fewer degrees than the corresponding MOP $g_{6}(\cdot)$. To see why, $g_{6 c}(\cdot)$ is computed as described in Eq. (6). $g_{1}\left(x_{1}\right)$ is a 2-piece, 3-degree MOP and $g_{5 c}\left(x_{1}, x_{3}\right)$ is a 6-piece, 3 -degree MOP. After multiplication, $g_{1}\left(x_{1}\right) g_{5 c}\left(x_{1}, x_{3}\right)$ is a 8-piece, 6-degree MOP defined on hypercubes. After integration with respect to $x_{1}$, the degrees associated with $x_{1}$ disappear, and three of the pieces do, resulting in a 6 -piece, 3 -degree MOP $g_{4 c}\left(x_{3}\right)$. Two of the six pieces are defined on singleton regions $\left(x_{3}=9\right.$ and 15$)$. On the other hand, $g_{6}(\cdot)$ is defined as in Eq. (12). $g_{1}\left(x_{1}\right)$ is a 2-piece, 3 -degree MOP, and $g_{2}\left(x_{1}, x_{3}-x_{1}\right)$ is a 2-piece, 3-degree MOP defined on hyper-rhombus. After multiplication, $g_{1}\left(x_{1}\right) g_{2}\left(x_{1}, x_{3}-x_{1}\right)$ is a 4-piece, 6-degree MOP defined on hyper-rhombus regions. After integration with respect to $x_{1}$, the result is a 9-piece, 7 -degree MOP. One of the nine pieces is defined on a singleton point $\left(x_{3}=15\right)$.

In summary, although mixed-tree hypercube MOPs initially require more pieces than hyper-rhombus MOPs, after integration, MOPs defined on hypercubes tend to lose pieces and lose degrees, whereas MOPs defined on hyper-rhombuses tend to increase pieces (some of these are defined on lower dimensional regions), and increase degrees.

Computation Time. Next, consider the times required for the computation of the marginals of $X_{2}$ and $X_{3}$ displayed in Table 2. Notice that the time required is a random variable. We repeated the experiment 10 times under identical conditions for both cases and computed the mean and standard error (SE) of the mean. The 97.5 percentile $t$-statistic is 2.25 and a $95 \%$ confidence interval for the mean is mean $\pm 2.25 \mathrm{SE}$.

Table 2: Times required for the computation of the marginals of $X_{2}$ and $X_{3}$.

\begin{tabular}{l|cc} 
Mean time in seconds (SE) & Hypercube & Hyper-rhombus \\
\hline Marg. PDF of $X_{2}$ & 1.42 & 3.33 \\
$g_{4 c}$ vs. $g_{4}$ & $(0.01)$ & $(0.01)$ \\
\hline Marg. PDF of $X_{3}$ & 1.35 & 4.92 \\
$g_{6 c}$ vs. $g_{6}$ & $(0.009)$ & $(0.003)$
\end{tabular}

Integrating a MOP defined on hypercube regions is faster than integrating a MOP defined on hyper-rhombus regions. For the latter, we have to first solve linear inequalities and then do the integration where the limits of integrations are the solutions of the inequalities.

Accuracy. Finally, consider the accuracy of the computed marginals for $X_{2}$ and $X_{3}$ in terms of KL-divergence, maximum absolute deviation, 
absolute error in the mean and absolute error in the variance, shown in Table 3 .

Hyper-rhombus MOPs are more accurate than mixed-tree hypercube MOPs by two orders of magnitude or more. We can increase accuracy of mixed-tree hypercube MOPs by increasing \# mixed-tree points (up to a point), but this will increase sizes and computation time. An important point is that in the case of mixed-tree hypercube MOPs, we have a choice of accuracy vs. computation time/sizes, which we don't with hyper-rhombuses.

Table 3: Accuracy of the computed marginals for $X_{2}$ and $X_{3}$.

\begin{tabular}{l|l|l} 
Accuracy & Hypercube & Hyper-rhombus \\
\hline Marg. PDF of $X_{2}$ & KL: 0.0082 & KL: 0.00002 \\
$g_{4 c}$ vs. $g_{4}$ & MAD: 0.0092 & MAD: 0.00004 \\
& AEM: 0.0 & AEM: 0.0 \\
& AEV: 1.1653 & AEV: 0.0188 \\
\hline Marg. PDF of $X_{3}$ & KL: 0.0480 & KL: 0.00003 \\
$g_{6 c}$ vs. $g_{6}$ & MAD: 0.0360 & MAD: 0.00004 \\
& AEM: 0.0 & AEM: 0.0 \\
& AEV: 2.6735 & AEV: 0.0284
\end{tabular}

\section{Re-approximation of MOPs/MTEs}

As we saw in the preceding section, in the process of integrating MOPs using convolutions, we may get pieces defined on lower-dimensional regions, which have no probabilities associated with them. For example, $g_{6}\left(x_{3}\right)$ is a 11-piece, 7-degree MOP. Three of the eleven pieces are defined on singleton regions, and these pieces have no probabilities associated with them. By re-approximating MOPs, we can reduce \# pieces and degrees (\# pieces and terms for MTEs), which will increase computational efficiency at a small cost in accuracy. We will describe two methods for re-approximating potentials. The first method is applicable both for MOPs and MTEs, whilst the second re-estimates the parameters of the potentials in a different way for MOPs and for MTEs.

\subsection{Re-approximation by Dropping Pieces}

A simple method of re-approximation is simply dropping pieces that are defined on lower-dimensional regions. Since there are no probabilities associated with such pieces, dropping these pieces causes no additional loss of accuracy. In some cases, we get pieces that have very low probabilities associated with them. In this case, we can drop such low probability 
pieces. One should make sure that the total probability associated with the dropped pieces stays below some limit (e.g., 0.05) so that the loss of accuracy of computation is not much. It is necessary to re-normalize the potentials after dropping pieces with positive probabilities.

In Sec. 5, we describe some re-approximations of some intermediate functions obtained while solving a sample hybrid Bayesian network using MOPs and MTEs. Notice that this method cannot be used to lower the degree of a MOP potential, or the number of exponential terms in a MTE potential.

\subsection{Re-approximation of MOPs using LIP with Chebyshev points}

Another method for re-approximating MOP potentials is by using LIPs (Lagrange Interpolating Polynomials) with Chebyshev points. ${ }^{12}$ To illustrate this, consider $g_{6}(\cdot)$, the marginal PDF of $X_{3}$ from the example in Section 3.3.2, which is defined on non-singleton intervals $(0,6),(6,9),(9,12]$, $(12,15),(15,18],(18,21),(21,24),(24,30)$. Let's re-approximate $g_{6}(\cdot)$ using 5 pieces as follows: $(0,6),[6,12),[12,18),[18,24),[24,30)$. Currently, we do not have a theory for the choice of pieces. Our strategy is to use fewer pieces by merging pieces of the MOP being approximated, and to keep the sizes of the pieces as equal as possible.

For each interval, we compute the Chebyshev points starting with a number of points $n$ initially set to a small number. A good starting choice is $n=4$. We compute the 3-degree LIP that passes through these 4-points. We need to verify that the LIP is non-negative on the interval. If not, we increase $n$. If the function being approximated is strictly positive over the interval, then we are guaranteed to find an interpolating polynomial that is non-negative for some $n$. This is because when we increase the number of Chebyshev points by 1 , the maximum error between the LIP and the polynomial being approximated is reduced by a factor of 2 . If the smallest $n$ that results in a polynomial that is non-negative is too high, we reduce the width of the interval (i.e., use more pieces) and re-start.

For approximating $g_{6}(\cdot)$, using $n=5$ for all five pieces results in a MOP that is non-negative on all the five pieces. Next we normalize the resulting 5-piece, 4-degree MOP so the total area under the MOP is 1 . Let $g_{6 r}(\cdot)$ denote the 5-piece, 4-degree MOP approximation of $g_{6}$ found using the above procedure. The accuracy measures of $g_{6 r}(\cdot)$ compared to $g_{6}(\cdot)$, of $g_{6 r}$ compared to $f_{X_{3}}^{\prime}$, the exact PDF of $X_{3}$ truncated to $(0,30)$, and of $g_{6}$ compared to $f_{X_{3}}^{\prime}$, the exact PDF of $X_{3}$ truncated to $(0,30)$ are shown in Table 4 . The comparison suggests that $g_{6 r}$ approximates $f_{X_{3}}^{\prime}$ similarly to $g_{6}$, and we conclude that $g_{6 r}(\cdot)$ is a good approximation of $g_{6}$.

The LIP method applies also for two or higher dimensional functions. There also exists Chebyshev points theory for two-dimensional regions. ${ }^{19}$ For regions in 3 or higher dimensions, we can use some extensions of the 
Table 4: Accuracy of the approximations of the marginal PDF of $X_{3}$ using the LIP method.

\begin{tabular}{l|cccc} 
Approximations & $K L$ & $M A D$ & $A E M$ & $A E V$ \\
\hline$g_{6 r}(\cdot)$ vs. $g_{6}(\cdot)$ & 0.00004 & 0.00003 & 0.0 & 0.0013 \\
$g_{6 r}$ vs. $f_{X_{3}}^{\prime}$ & 0.00005 & 0.00004 & 0.0 & 0.0297 \\
$g_{6}$ vs. $f_{X_{3}}^{\prime}$ & 0.00003 & 0.00004 & 0.0 & 0.0284
\end{tabular}

one-dimensional Chebyshev point theory. One problem with using LIP with Chebyshev points in two or higher dimensions is non-negativity. It may be necessary to increase the degree of the MOP potential to a very high number making computations numerically unstable. In such cases, we can resort to the idea behind mixed-tree approximations, and use a one-dimensional LIP method with Chebyshev points to approximate a two or higher dimensional function. In Sec. 5, we describe a re-approximation of a MOP (using a one-dimensional LIP method with Chebyshev points) that is obtained as an intermediate function while solving a sample hybrid BN using MOPs defined on hypercubes.

\subsection{Re-approximation of MTEs Using Numeric Least Squares}

We discuss how to re-approximate MTEs by reducing the number of pieces and exponential terms. The idea behind the re-approximation method is similar to the one used for MOPs, but using a different mathematical tool. In this case, we rely on the Levenberg-Marquardt (LM) algorithm, ${ }^{20,21}$ available via the command FindFit provided by Mathematica ${ }^{\circledR}$. The LM algorithm is for minimizing the sum of the squares of the deviations of the fitted model from the exact one. It is controlled by a so-called damping parameter, $\lambda$, which is automatically adjusted in each iteration. If the reduction in the sum of squares is fast, $\lambda$ is set to a small value and the LM algorithm becomes similar to the Gauss-Newton method. Otherwise, $\lambda$ is set to a high value, and the LM algorithm becomes closer to a gradient descent method. Selecting appropriate starting values for the set of parameters to estimate is an important issue in order to avoid local optima. Since the MTE approximation to the Gaussian distribution is quite accurate, we will use the parameter estimates of this approximation as starting values for the LM algorithm. The steps to re-approximate an MTE density $f$ for a given partition of the domain, and a fixed number of terms are detailed in Algorithm 1 .

Note that this procedure is flexible. For example, if the shape of the function to re-approximate is not too irregular (for instance, if it has smooth parts), we can set a different number of terms in different regions, with fewer 


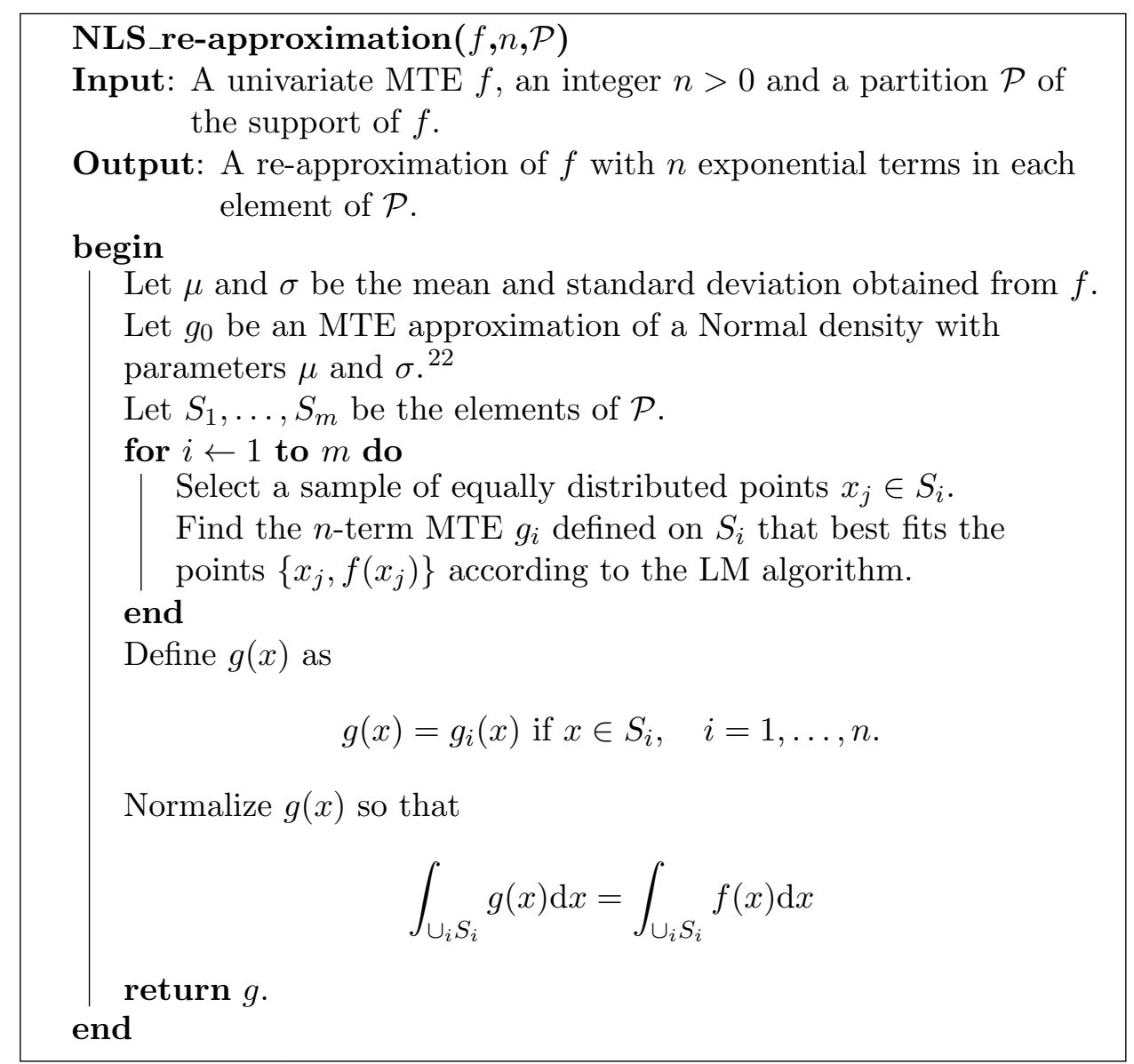

Algorithm 1: An algorithm for re-approximating MTEs using the LM method.

terms in the more uniform ones. Similar to MOPs, there is not yet a formal method to divide the domain. We followed the strategy of splitting the domain if the shape of the function differs from Gaussian shape. This is also related to a successful selection of the starting points, as it requires that the density in the piece being re-approximated somehow resembles a Gaussian shape, which is not always the case. Another possible strategy for splitting the domain, already explored in the literature ${ }^{23}$ consists of choosing points corresponding to extremes and inflection points. Such heuristic is appropriate when the number of exponential terms is low, but when the number of exponential terms is allowed to be higher, splitting by those points my be useless, as the MTE function is able to accurately represent changes in inflection and extremes. ${ }^{13}$

In the case of re-approximating 2 or higher dimensional potentials, we use the same procedure as MOPs, i.e. using mixed trees and fixing one dimension whilst re-approximating the function for the remaining dimensions. In the 
case of MTEs this is not difficult, since this is the approach selected to define the conditional distributions (parent variables can only affect the partition of the domain, not the expression of the density itself).

Note that the re-approximation technique can lead to very accurate solutions, in which the complexity reduction is minimal, or to very smooth approximations, but with a big decrease in the complexity. It depends on the number of pieces selected and the number of exponential terms included (or degree of the polynomial in the MOP case). Finding a tradeoff between these two extremes is still an open question. This issue has been addressed from the point of view of learning, when a data sample is available ${ }^{24}$ proposing a heuristic based on finding the configuration that maximizes the Bayes information criterion (BIC) score of the model.

\subsection{Experimental evaluation}

In order to evaluate how the complexity of inference grows and how our proposed solution scales up, we have conducted a series of experiments over networks of increasing complexity as depicted in Figure 5. The experiments consisted of computing the marginal for $Y_{1}$ in network (a) and $Y_{2}$ in (b). The initial distributions in the networks are such that $X_{1} \sim \mathcal{N}(0,1), X_{i} \mid x_{i-1} \sim$ $\mathcal{N}\left(x_{i-1}, 1\right), i=2,3, Y_{1}=X_{1}+X_{2}$ and $Y_{2}=Y_{1}+X_{3}$. Therefore, we have deterministic conditionals for $Y_{1}, Y_{2}$.

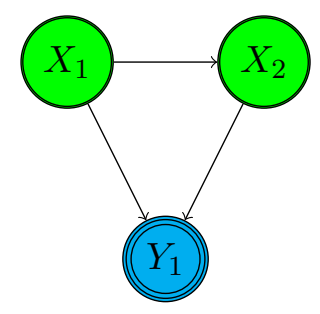

(a)

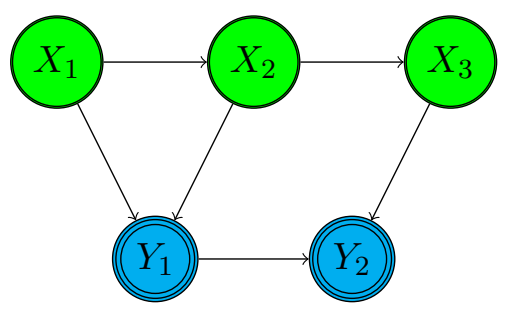

(b)

Figure 5: Networks used in the experimental evaluation.

We attempted to evaluate each network using the next three schemes:

- Hyper-rhombus MOPs. In this case, no approximations are carried out. Only pieces that correspond to singletons (and that, therefore, have null probability allocated) are removed.

- Hypercube MOPs with 3 pieces per variable. In this approach, when the hypercube is lost, re-approximation using LIPs with Chebyshev points is employed. 
- Hypercube MTEs with 3 pieces per variable. Similarly, when the hypercube condition is lost, re-approximation using numerical least squares is applied.

Table 5: Results of the experiments carried out over the networks in Figure 5. The exact mean and variance for network (a) are $\mu=0, \sigma^{2}=5.76$, while for (b) these values are $\mu=0, \sigma^{2}=13.9995$. Note that Mathematica ${ }^{\circledR}$ is unable to compute the KL divergence for the estimated MTEs.

\begin{tabular}{cll|ccccccc} 
Net & Model & Domain & $\hat{\mu}$ & $\hat{\sigma}^{2}$ & $K L$ & $M A D$ & $A E M$ & $A E V$ & Time \\
\hline (a) & MOP & Hyper-cube & 0 & 5.44 & 0.073458 & 0.0806 & 0 & 0.3208 & 412.84 \\
(a) & MOP & Hyper-rhombus & 0 & 4.87 & 0.013378 & 0.01651 & 0 & 1.10387 & 47.47 \\
(b) & MOP & Hyper-rhombus & 0 & 13.65 & 0.00041 & 0.00069 & 0 & 0.348 & 672.44 \\
(a) & MTE & Hyper-cube & -0.0008 & 5.1296 & -- & 0.1049 & 0.000822 & 0.638831 & 1181.82 \\
(b) & MTE & Hyper-cube & -0.00158 & 16.07 & -- & 0.04883 & 0.00158 & 2.071 & 3273.65
\end{tabular}

The results of the experiments are shown in Table 5 , where for each estimated model we have measured the mean and variance $\left(\hat{\mu}, \hat{\sigma}^{2}\right)$, maximum absolute deviation $(M A D)$, absolute error in the mean $(A E M)$, absolute error in the variance $(A E V)$ and computing time. Note that, even though MOPs with hyper-rhombuses do not carry out approximations during inference, it underestimates the variance due to the fact that, in this experiment, we consider Gaussian variables, whose domain is infinite, while MOPs and MTEs have finite domain by definition.

In what concerns the growth of the complexity of inference, computing time of MOPs with hyper-rhombuses (i.e. with no approximation) is over 10 times higher in network (b) with respect to network (a). Note also that hyper-cube MOP for network (a) is much more costly (close to 10 times more) than MOP hyper-rhmobuses. It indicates that the time required to interpolate the approximate densities using LIP is not negligible. Regarding MTEs, the added complexity due to the inclusion of $X_{3}$ and $Y_{2}$ in network (b) causes the execution time to be close to 3 times higher than in network (a). Adding an extra pair of variables $X_{4}, Y_{3}$ makes the problem intractable with any of the methods.

\section{Case Study: Solving a PERT Hybrid Bayesian Network}

We will illustrate the inference process in hybrid BNs using a stochastic PERT network. ${ }^{15}$ This problem has previously been addressed using BNs, ${ }^{25}$ but the approach we propose here is more flexible and general, as it allows to compute the full distribution of the variables of interest, and not 


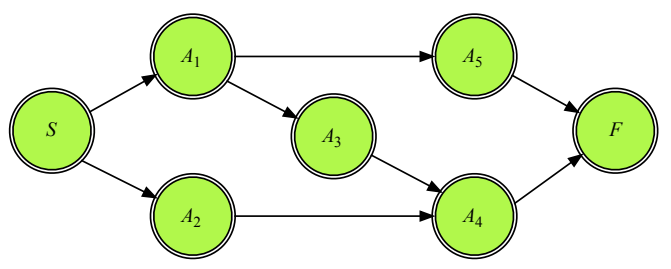

Figure 6: A PERT network with five activities

only their expectations and variances. PERT stands for Program Evaluation and Review Technique, and is one of the commonly used project management techniques. ${ }^{26}$ PERT networks are directed acyclic networks where the nodes represent duration of activities and the arcs represent precedence constraints in the sense that before we can start any activity, all the parent activities have to be completed. The term stochastic refers to the fact that the duration of activities are modeled as continuous random variables. A previous proposal on using hybrid BNs for project scheduling under uncertainty is based on adapting the so-called critical path method to incorporate uncertainty, and representing the resulting model as a hybrid BN that is afterwards solved using dynamic discretization. ${ }^{27}$ Our solution sidesteps the discretization problem by directly employing MTEs and MOPs.

Figure 6 shows a PERT network with 5 activities $A_{1}, \ldots, A_{5}$. Nodes $S$ and $F$ represent the start and finish times of the project. The links among activities mean that an activity cannot be started until after all its predecessors have been completed. Assume we are informed that the durations of $A_{1}$ and $A_{3}$ are positively correlated, and the same is true with $A_{2}$ and $A_{4}$. Then, this PERT network can be transformed into a BN as follows.

Let $D_{i}$ and $C_{i}$ denote the duration and the completion time of the activity $i$, respectively. The activity nodes in the PERT network are replaced with activity completion times in the BN. Next, activity durations are added 


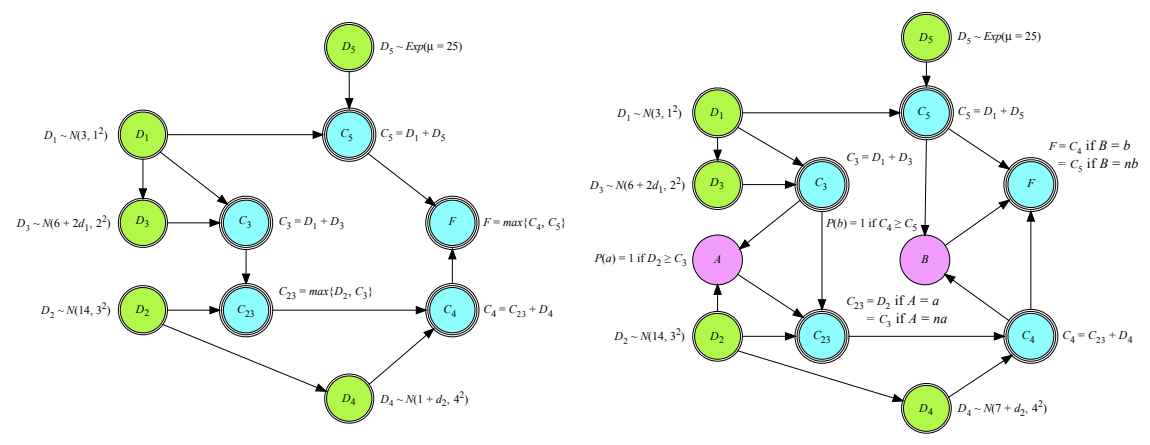

Figure 7: A BN (left) and a hybrid BN (right) representing the PERT network in Figure 6.

with a link from $D_{i}$ to $C_{i}$, so that each activity will be represented by two nodes, its duration $D_{i}$ and its completion time $C_{i}$. Notice that the completion times of the activities which do not have any predecessors will be the same as their durations. Hence, activities $A_{1}$ and $A_{2}$ will be represented just by their durations, $D_{1}$ and $D_{2}$. As $A_{3}$ and $A_{1}$ have positively correlated durations, a link will connect $D_{1}$ and $D_{3}$ in the BN. For the same reason, another link will connect $D_{2}$ and $D_{4}$. The completion time of $A_{3}$ is $C_{3}=D_{1}+D_{3}$. Let $C_{23}=\max \left\{D_{2}, C_{3}\right\}$ denote the completion time of activities $A_{2}$ and $A_{3}$. The completion time of activity $A_{5}$ is $C_{5}=D_{1}+D_{5}$, and for activity $A_{4}$, it is $C_{4}=C_{23}+D_{4}$.

We assume that the project start time is zero and each activity is started as soon as all the preceding activities are completed. Accordingly, $F$ represents the completion time of the project, which is the maximum of $C_{5}$ and $C_{4}$. The resulting PERT BN is given in Figure 7 (left).

Notice that the conditionals for the variables $C_{3}, C_{23}, C_{4}, C_{5}$ and $F$ are deterministic, in the sense that their conditional distributions given their parents have zero variances. On the other hand, variables $D_{1}, \ldots, D_{5}$ are continuous random variables, and their corresponding conditional distributions are depicted next to their corresponding nodes in Figure 7. The parameters $\mu$ (mean) and $\sigma^{2}$ (variance) of the Normal distribution are in units of days and days ${ }^{2}$, respectively. The parameter $\mu$ (mean) of the exponential distribution is in units of days.

Using the re-approximation techniques described in Section 4, we were able to solve the PERT hybrid Bayesian network using MOPs defined on 
hypercubes and using MTEs. Using MOPs on hypercubes, evaluation of the entire Mathematica notebook (all computations including re-approximation) takes about 169.15 seconds (2.82 minutes). Using MTEs, evaluation of the entire Mathematica notebook (all computations including re-approximation) takes about 142.65 seconds (about 2.4 minutes). Details of all computations including re-approximations are given in the next sub-sections. Without re-approximations of some of the intermediate functions, we get a "lack of memory" warning message, even though the available memory was 16GBs. A reason for this is as follows. During the solution of the PERT hybrid Bayesian network problem, we get an intermediate function $f_{4}$ for $\left\{C_{3}, C_{5}\right\}$ that is a 23-piece, 8-degree MOP, and another intermediate function $f_{7}$ for $\left\{C_{3}, C_{4}\right\}$ that is a 17 -piece, 7 -degree MOP. In the process of marginalizing $C_{3}$, we have to multiply $f_{4}$ and $f_{7}$, and this results in a MOP that has up to 391 pieces and 17-degrees, and Mathematica runs out of memory during the marginalization process.

\subsection{Solution Using Hypercube MOPs}

We start with a 2-piece, 3-degree MOP $f_{1}(\cdot)$ as an approximation of the $\mathrm{PDF}$ of the standard normal. Using $f_{1}(\cdot)$, we define a 2-piece, 3-degree MOP approximation of the PDFs of $D_{1}$ and $D_{2}$. Using mixed trees, we define a 6 -piece, 3-degree MOP of the conditional PDFs of $D_{3}$ and $D_{4}$. We used a 2-piece, 3-degree MOP approximation of the $\operatorname{Exp}(25)$ density for $D_{5}$.

The initial potentials in the PERT hybrid BN in the right panel of Figure 7 are summarized in Table 6 .

Table 6: Summary of the initial potentials in the PERT hybrid BN. Type CD means conditional density and DC stands for deterministic conditional.

\begin{tabular}{l|c|c||l|l|c} 
Variable & Potential & Type & Variable & Potential & Type \\
\hline$D_{1}$ & $f_{D_{1}}$ & $\mathrm{CD}$ & $A$ & $p_{A a}\left(d_{2}, c_{3}\right)=P\left(A=a \mid d_{2}, c_{3}\right)$ & $\mathrm{CD}$ \\
& & & & $p_{A n a}\left(d_{2}, c_{3}\right)=P\left(A=n a \mid d_{2}, c_{3}\right)$ & \\
$D_{2}$ & $f_{D_{2}}$ & $\mathrm{CD}$ & $C_{3}$ & $f_{C_{3}}\left(d_{1}, d_{3}, c_{3}\right)=\delta\left(c_{3}-d_{1}-d_{3}\right)$ & $\mathrm{DC}$ \\
$D_{3}$ & $f_{D_{3}}$ & $\mathrm{CD}$ & $C_{4}$ & $f_{C_{4}}\left(c_{23}, c_{4}, d_{4}\right)=\delta\left(c_{4}-c_{23}-d_{4}\right)$ & $\mathrm{DC}$ \\
$D_{4}$ & $f_{D_{4}}$ & $\mathrm{CD}$ & $C_{5}$ & $f_{C_{5}}\left(d_{1}, d_{5}, c_{5}\right)=\delta\left(c_{5}-d_{1}-d_{5}\right)$ & $\mathrm{DC}$ \\
$D_{5}$ & $f_{D_{5}}$ & $\mathrm{CD}$ & $C_{23}$ & $f_{C_{23 a}}\left(d_{2}, c_{3}, c_{23}\right)=\delta\left(c_{23}-d_{2}\right)$ if $A=a$ & $\mathrm{DC}$ \\
& & & & $f_{C_{23 n a}}\left(d_{2}, c_{3}, c_{23}\right)=\delta\left(c_{23}-c_{3}\right)$ if $A=n a$ & \\
& & & $f_{F b}\left(c_{4}, c_{5}, f\right)=\delta\left(f-c_{4}\right)$ if $B=b$ & $\mathrm{DC}$ \\
& & & $f_{F n b}\left(c_{4}, c_{5}, f\right)=\delta\left(f-c_{5}\right)$ if $B=n b$ & \\
& & & $p_{B b}\left(c_{4}, c_{5}\right)=P\left(B=b \mid c_{4}, c_{5}\right)$ & $\mathrm{CD}$ \\
& & & & $p_{B n b}\left(c_{4}, c_{5}\right)=P\left(B=n b \mid c_{4}, c_{5}\right)$ &
\end{tabular}

The goal is to compute the marginal density for $F$. To that end, we choose an elimination order of the remaining variables in the network, namely 
$D_{5}, D_{3}, D_{1}, D_{4}, D_{2}, C_{23}, A, C_{3}, C_{4}, C_{5}$, and $B$. Different elimination orders can influence the complexity of the inference process. A discussion on the selection of the elimination order in hybrid BNs can be found in the related literature. ${ }^{9}$

Table 7: Potentials computed during the elimination of the variables in the PERT hybrid BN. The first column indicates the variables being eliminated, and the second contains the potentials obtained after eliminating them.

\begin{tabular}{|c|c|}
\hline Variable & Potential computed \\
\hline$D_{5}$ & $f_{2}\left(d_{1}, c_{5}\right)=\int_{-\infty}^{\infty} f_{D_{5}}\left(d_{5}\right) f_{C_{5}}\left(d_{1}, d_{5}, c_{5}\right) \mathrm{d} d_{5}=f_{D_{5}}\left(c_{5}-d_{1}\right)$ \\
\hline$D_{3}$ & $f_{2}\left(d_{1}, c_{5}\right)=\int_{-\infty}^{\infty} f_{D_{5}}\left(d_{5}\right) f_{C_{5}}\left(d_{1}, d_{5}, c_{5}\right) \mathrm{d} d_{5}=f_{D_{5}}\left(c_{5}-d_{1}\right)$ \\
\hline$D_{1}$ & $f_{4}\left(c_{3}, c_{5}\right)=\int_{-\infty}^{\infty} f_{D_{1}}\left(d_{1}\right) f_{2 c}\left(d_{1}, c_{5}\right) f_{3 c}\left(d_{1}, c_{3}\right) \mathrm{d} d_{1}$ \\
\hline$D_{4}$ & $f_{5}\left(c_{23}, d_{2}, c_{4}\right)=\int_{-\infty}^{\infty} f_{D_{4}}\left(d_{2}, d_{4}\right) f_{C_{4}}\left(c_{23}, c_{4}, d_{4}\right) \mathrm{d} d_{4}=f_{D_{4}}\left(d_{2}, c_{4}-c_{23}\right)$ \\
\hline$D_{2}$ & $\begin{aligned} f_{6 a}\left(c_{3}, c_{23}, c_{4}\right) & =\int_{-\infty}^{\infty} f_{D_{2}}\left(d_{2}\right) f_{C_{23 a}}\left(d_{2}, c_{3}, c_{23}\right) f_{5 c}\left(c_{23}, d_{2}, c_{4}\right) p_{A a}\left(d_{2}, c_{3}\right) \mathrm{d} d_{2} \\
& =f_{D_{2}}\left(c_{23}\right) f_{5 c}\left(c_{23}, c_{23}, c_{4}\right) p_{A a}\left(c_{23}, c_{3}\right) \\
f_{6 n a}\left(c_{3}, c_{23}, c_{4}\right) & =f_{C_{23 n a}}\left(d_{2}, c_{3}, c_{23}\right) \int_{-\infty}^{\infty} f_{D_{2}}\left(d_{2}\right) f_{5 c}\left(c_{23}, d_{2}, c_{4}\right) p_{A n a}\left(d_{2}, c_{3}\right) \mathrm{d} d_{2} \\
& =\delta\left(c_{23}-c_{3}\right) \int_{-\infty}^{c_{3}} f_{D_{2}}\left(d_{2}\right) f_{5 c}\left(c_{23}, d_{2}, c_{4}\right) \mathrm{d} d_{2}\end{aligned}$ \\
\hline$C_{23}$ & $\begin{aligned} f_{7 a}\left(c_{3}, c_{4}\right) & =\int_{-\infty}^{\infty} f_{6 a}\left(c_{3}, c_{23}, c_{4}\right) \mathrm{d} c_{23} \\
& =\int_{-\infty}^{\infty} f_{D_{2}}\left(c_{23}\right) f_{5}\left(c_{23}, c_{23}, c_{4}\right) p_{A a}\left(c_{23}, c_{3}\right) \mathrm{d} c_{23} \\
& =\int_{c_{3}}^{\infty} f_{D_{2}}\left(c_{23}\right) f_{5}\left(c_{23}, c_{23}, c_{4}\right) \mathrm{d} c_{23} \\
f_{7 n a}\left(c_{3}, c_{4}\right) & =\int_{-\infty}^{\infty} f_{6 n a}\left(c_{3}, c_{23}, c_{4}\right) \mathrm{d} c_{23}=\int_{-\infty}^{c_{3}} f_{D_{2}}\left(d_{2}\right) f_{5}\left(c_{3}, d_{2}, c_{4}\right) \mathrm{d} d_{2}\end{aligned}$ \\
\hline$A$ & $f_{7}\left(c_{3}, c_{4}\right)=f_{7 a}\left(c_{3}, c_{4}\right)+f_{7 n a}\left(c_{3}, c_{4}\right)$ \\
\hline$C_{3}$ & $f_{8}\left(c_{4}, c_{5}\right)=\int_{-\infty}^{\infty} f_{4 r}\left(c_{3}, c_{5}\right) f_{7 r}\left(c_{3}, c_{4}\right) \mathrm{d} c_{3}$ \\
\hline$C_{4}$ & $\begin{aligned} f_{9 b}\left(c_{5}, f\right) & =\int_{-\infty}^{\infty} p_{B b}\left(c_{4}, c_{5}\right) f_{F b}\left(c_{4}, c_{5}, f\right) f_{8}\left(c_{4}, c_{5}\right) \mathrm{d} c_{4} \\
& =\int_{-\infty}^{\infty} p_{B b}\left(c_{4}, c_{5}\right) \delta\left(f-c_{4}\right) f_{8}\left(c_{4}, c_{5}\right) \mathrm{d} c_{4} \\
& =p_{B b}\left(f, c_{5}\right) f_{8}\left(f, c_{5}\right) \\
f_{9 n b}\left(c_{5}, f\right) & =\int_{-\infty}^{\infty} f_{F n b}\left(c_{4}, c_{5}, f\right) p_{B n b}\left(c_{4}, c_{5}\right) f_{8}\left(c_{4}, c_{5}\right) \mathrm{d} c_{4} \\
& =\delta\left(f-c_{5}\right) \int_{-\infty}^{c_{5}} f_{8}\left(c_{4}, c_{5}\right) \mathrm{d} c_{4}\end{aligned}$ \\
\hline$C_{5}$ & $\begin{array}{l}f_{10 b}(f)=\int_{-\infty}^{\infty} f_{9 b}\left(c_{5}, f\right) \mathrm{d} c_{5}=\int_{-\infty}^{f} f_{8}\left(f, c_{5}\right) \mathrm{d} c_{5} \\
f_{10 n b}(f)=\int_{-\infty}^{\infty} f_{9 n b}\left(c_{5}, f\right) \mathrm{d} c_{5}=\int_{-\infty}^{f} f_{8}\left(c_{4}, f\right) \mathrm{d} c_{4}\end{array}$ \\
\hline$B$ & $f_{11}(f)=f_{10 b}(f)+f_{10 n b}(f)$ \\
\hline
\end{tabular}

The potentials obtained after eliminating each variable are shown in Table 7. The deletion of $D_{5}$ is carried out by computing $f_{2}\left(d_{1}, c_{5}\right)$. Notice that $f_{2}$ is not defined on hypercubes. So we approximate $f_{2}$ by $f_{2 c}$ by using a 3-point mixed tree approximation. Next we delete $D_{3}$ by computing $f_{3}\left(d_{1}, c_{3}\right)$. Notice that $f_{3}$ is not defined on hypercubes. So we approximate $f_{3}$ by $f_{3 c}$ by using a 3 -point mixed tree approximation.

Next we delete $D_{1}$ by computing $f_{4}\left(c_{3}, c_{5}\right)$. It is computed as a 23piece, 8-degree MOP, and takes 5.05 seconds to be calculated. Next, we re-approximate $f_{4}$ by $f_{4 r}$, as follows. 9 of the 23 pieces are defined on 1-dimensional regions. Thus, we can safely drop these pieces resulting in a 14-piece MOP. Further examination of these 14 pieces reveals that 6 of 

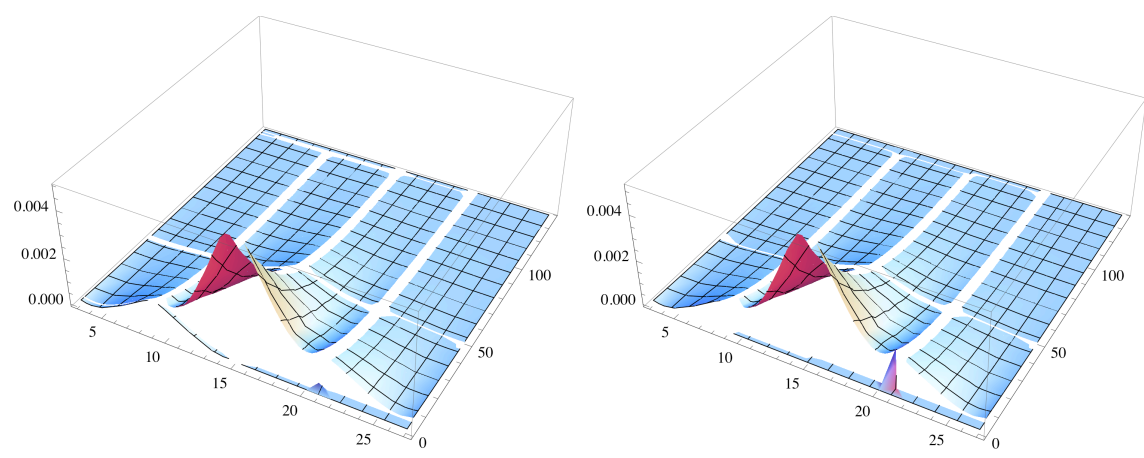

Figure 8: 3-D plots of $f_{4}$ (left) and $f_{4 r}$ (right).

the pieces have very small probabilities (0.000017 to 0.026$)$ associated with them. The total probability associated with these 6 pieces is $\approx 0.044$. After we drop these 6 pieces and re-normalize the potential, we obtain a 8-piece MOP $f_{4 r}\left(c_{3}, c_{5}\right)$ that can be used in place of $f_{4}\left(c_{3}, c_{5}\right)$. Figure 8 shows 3-D plots of $f_{4}$ and $f_{4 r}$.

Next, we delete $D_{4}$ by computing $f_{5}\left(c_{23}, d_{2}, c_{4}\right)$, which is not defined on hypercubes. As before, we re-define $f_{5}$ by $f_{5 c}$, which is defined on a 3 -point mixed tree hypercube.

Next, the deletion of $D_{2}$ yields the functions $f_{6 a}\left(c_{3}, c_{23}, c_{4}\right)$ and $f_{6 n a}\left(c_{3}, c_{23}, c_{4}\right)$. Afterwards, we marginalize out $C_{23}$ obtaining $f_{7 a}\left(c_{3}, c_{4}\right)$ and $f_{7 n a}\left(c_{3}, c_{4}\right)$.

Next we delete $A$ by computing $f_{7}\left(c_{3}, c_{4}\right)$. Potential $f_{7}$ is computed as a 17-piece, 7-degree MOP and it requires 16.82 seconds to be computed. Given the large number of pieces, which may lead to unmanageable functions after a new multiplication, we re-approximate $f_{7}$ by $f_{7 r}$, an 8-piece, 7 -degree MOP using LIPs with Chebyshev points as follows.

$f_{7}$ is computed on the domain $\left(3<c_{3}<27\right) \times\left(1.125<c_{5}<137.5\right)$. We will approximate this potential by a 8-piece, 7-degree MOP $f_{7 r}$ as follows. Consider a 4 -way partition of $\left(3<c_{3}<27\right)$ as follows: $(3,11),[11,17)$, $[17,23)$, and $[23,27)$. Consider the region $3<c_{3}<11$. We approximate $f_{7}\left(7, c_{4}\right)$ (a 1 -dimensional function, $c_{3}=7$ is the mid-point of the interval) using LIP with Chebyshev points as discussed earlier by a 2-piece, 7-degree MOP and use this approximation for the region $\left(3<c_{3}<11\right) \times\left(11<c_{5}<\right.$ 59 ). By doing this for all four pieces of the partition of the domain of $C_{3}$, we obtain a 8-piece, 7 -degree MOP approximation $f_{7 r}$ of the two-dimensional MOP $f_{7}$. Figure 9 shows 3 -D plots of $f_{7}$ and $f_{7 r}$.

Next, after elimination of $C_{3}, f_{8}\left(c_{4}, c_{5}\right)$ is computed as a 53-piece, 10degree MOP in 26.01 seconds. The elimination of $C_{4}$ consists of computing $f_{9 b}\left(c_{5}, f\right)$ and $f_{9 n b}\left(c_{5}, f\right)$. The elimination of $C_{5}$ takes 108.19 seconds, required to calculate $f_{10 b}(f)$ and $f_{10 n b}(f)$.

Finally, by eliminating $B$, we obtain $f_{11}(f)$, which is a 22-piece, 11- 

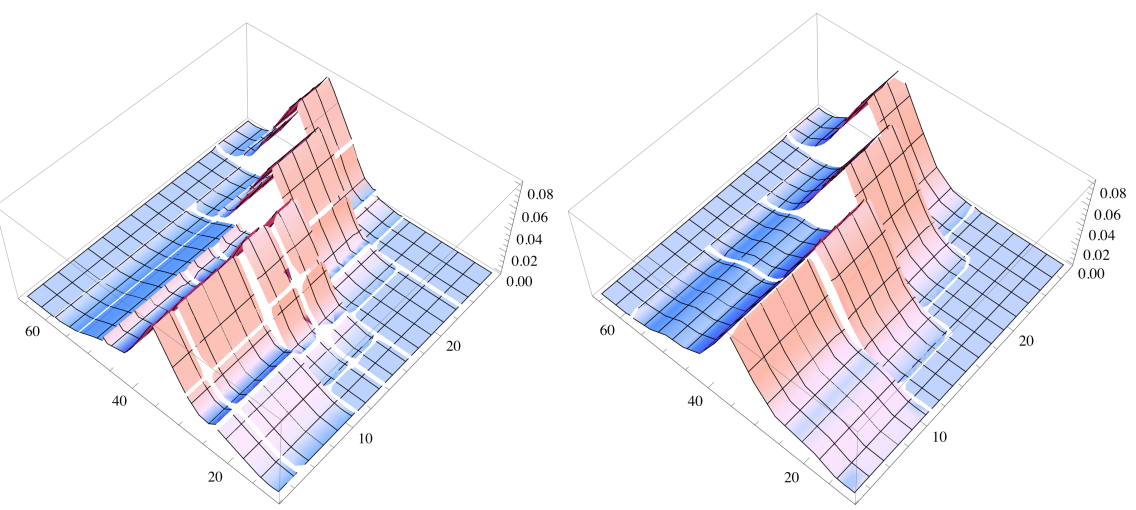

Figure 9: 3-D plots of $f_{7}$ (left) and $f_{7 r}$ (right).

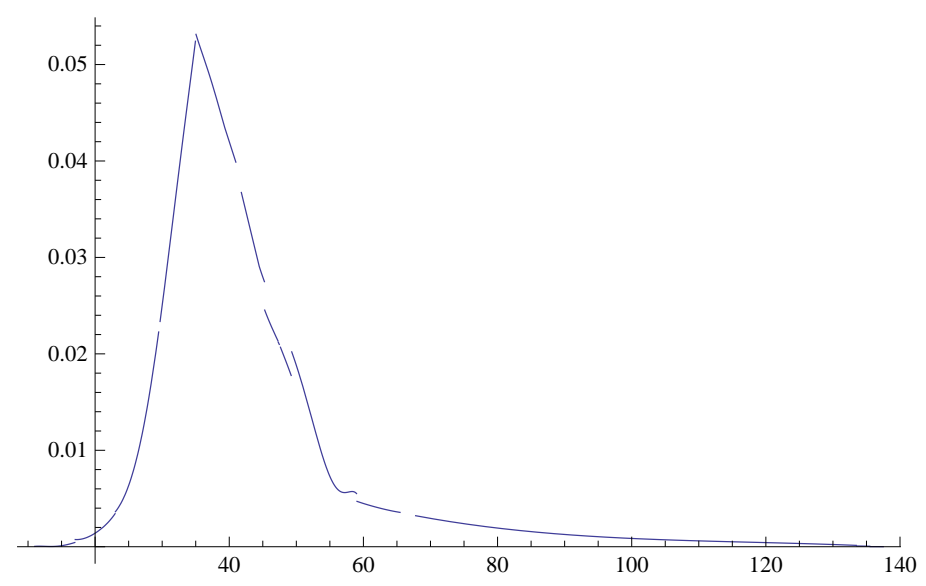

Figure 10: The MOP approximation $f_{11}$ of the marginal of $F$.

degree MOP representation of the PDF of $F$. Using $f_{11}$, we compute the expectation and standard deviation of $F: \mu_{F}=43.44$ days and $\sigma_{F}=15.71$ days, respectively. A plot of $f_{11}$ is shown in Figure 10. Evaluation of the entire Mathematica notebook (all computations including re-approximation) takes about 169.15 seconds (2.8 minutes).

\subsection{Solution using MTEs}

The inference process to solve the PERT network using MTEs is exactly the same as in the previous section using MOPs, but using MTE potentials instead. Therefore, we will only describe here the differences about the sizes of the potentials and the computations times, as well as the result obtained.

In Section 3.1, we explained how to avoid the problem of obtaining nonMTE potentials when doing the convolution operation. However, there is another problem when dealing with the $\max$ operations involved in the net- 
Table 8: Parameter values of the MTE approximation to the Gaussian distribution.

\begin{tabular}{c|cccccc}
$a_{i}$ & 73750.9 & -235407 & 146016 & 146855 & -140195 & 8980.02 \\
\hline$b_{i}$ & -0.045451 & -0.027365 & -0.006084 & -0.006084 & 0.013520 & 0.065411
\end{tabular}

work, in particular when computing $f_{7 a}, f_{7 n a}, f_{10 b}$, and $f_{10 n b}$. In all those cases, because the probability potentials for discrete variables $A$ and $B$ are not defined on hypercubes, one of the integration limits is a variable, which yields a non-MTE potential. As an example illustrating this issue consider an MTE potential as follows:

$$
f(x, y)=1+e^{3 x+2 y} \quad \text { if } 3 \leq x \leq 5,1 \leq y \leq 5, \text { and } x \geq y .
$$

Integrating out $y$ we obtain

$$
\int_{1}^{x} f(x, y) d y=x-1+\frac{1}{2} e^{5 x}-\frac{1}{2} e^{3 x+2}, \quad \text { if } 3 \leq x \leq 5,
$$

which is not an MTE function, as it contains a polynomial term. In this case, the problem is due to the constant term $a_{0}=1$ in $f(x, y)$, and it can be avoided by not including it in Definition 1 of an MTE function. Using this version of MTEs, the PERT network can be solved within the MTE class. Thus, instead of using the original definition, for solving the PERT network we use

$$
f(\mathbf{z})=\sum_{i=1}^{m} a_{i} \exp \left\{\mathbf{b}_{i}^{\top} \mathbf{z}\right\}
$$

in all the MTE potentials involved in the solution.

So, we start with a 1-piece, 6-terms (without a constant term) MTE, $f_{1}(\cdot)$, as an approximation of the PDF of the standard normal PDF on the domain $[-2.5,2.5]$ (see the parameters in Table 8), obtained using the LM algorithm (see Section 4.3) and using as initial values the ones obtained by Langseth et al. ${ }^{22}$ in their approximation of the PDF of the standard normal density. Using $f_{1}(\cdot)$, we define a 1-piece, 6-terms MTE approximation of the PDF of $D_{1}$ and $D_{2}$. Using mixed trees, we define a 2-piece, 6-terms MTE approximation of the conditional PDFs of $D_{3}$ and $D_{4}$. Since the PDF of the exponential distribution is already a 1-piece, 1-term MTE, no approximation is needed for the PDF of $D_{5}$.

In order to compute the marginal for $F$, we use the same elimination order as for the case of MOPs. After the elimination of $D_{5}, D_{3}$, and $D_{1}$, we compute a 11-piece, 6-terms MTE $f_{4}\left(c_{3}, c_{5}\right)$ in 4.54 seconds. We reapproximate $f_{4}$ as follows.

One of the 11 pieces is a singleton point, which due to the continuous nature of the distribution can be excluded. Three of the remaining pieces have very low probabilities (0.00005 to 0.019$)$ with a total mass of 0.02594 . 
After dropping these pieces and re-normalizing, the density is a 4-piece MTE $f_{4 s}\left(c_{3}, c_{5}\right)$ that can be used in place of $f_{4}\left(c_{3}, c_{5}\right)$. Figure 11 shows $f_{4}$ and $f_{4 s}$.

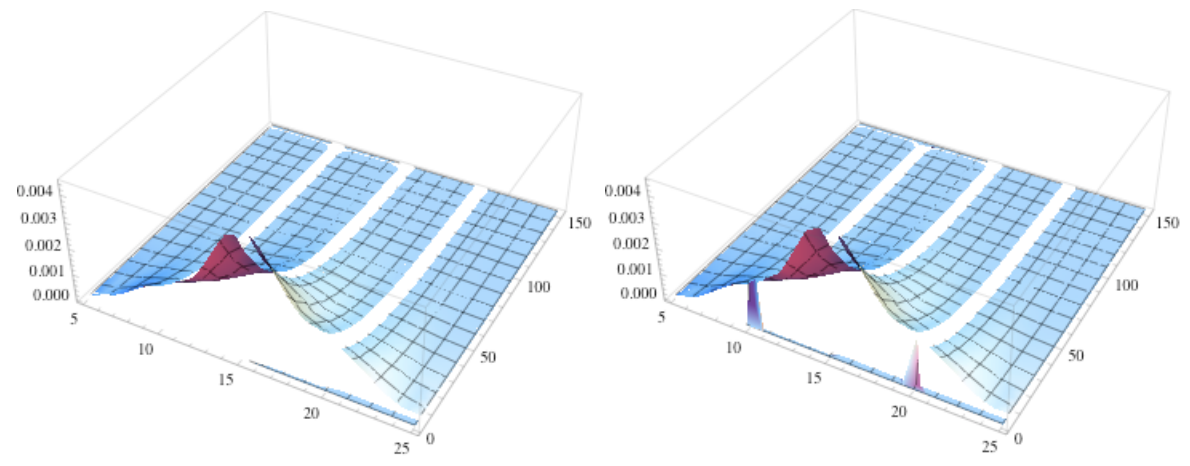

Figure 11: MTEs corresponding to $f_{4}$ (left) and $f_{4 s}$ (right).

After further elimination of $D_{4}, D_{2}, C_{23}$ and $A$, we compute a 16-piece, 72 -terms MTE $f_{7}\left(c_{3}, c_{4}\right)$ in 31.96 seconds, which we re-approximate as a 7 -piece potential $f_{7 s}$, with 6 terms for 5 of the pieces and 2 terms for the remaining 3 pieces.

After further elimination of $C_{3}$, we obtain a 20-piece, and between 4 and 10 terms MTE $f_{8}\left(c_{4}, c_{5}\right)$ in 29.21 seconds. Then we delete $C_{4}$, and $C_{5}$ (need 59.14 seconds), and $B$, obtaining a 5 -piece, and between 2 and 38 terms MTE approximation of the marginal PDF of $F$. A graph of it is displayed in Figure 12. We compute the expectation and standard deviation: $E(F)=43.79$ days and $\sigma_{F}=16.17$ days, respectively. The evaluation of the entire Mathematica notebook (all computations) takes about 142.65 seconds (about 2.4 minutes).

\subsection{Solution Using Simulation}

In order to have a clearer idea of the true marginal for $F$, we computed an estimate of it by simulating a sample of size $n=1,000,000$ of $D_{1}, \ldots, D_{5}$ using plain Monte Carlo simulation and then computing the corresponding values of $C_{3}, C_{23}, C_{4}, C_{5}$ and $F$ for each record in the sample, according to their definition. Then, we fitted a Gaussian kernel density to the values obtained for $F$. The result is displayed in Figure 13. In this case, point estimates of the expectation and standard deviation of $F$ are $\hat{E}(F)=36.19$ and $\hat{\sigma}_{F}=20.28$ days, respectively.

We have also computed confidence intervals for both parameters, using the statistic $\frac{\bar{X}-\mu}{s / \sqrt{n}} \sim t(n-1)$ for the mean and the statistic $\frac{(n-1) S^{2}}{\sigma^{2}} \sim$ $\chi^{2}(n-1)$ for the standard deviation. The obtained $95 \%$ confidence interval 


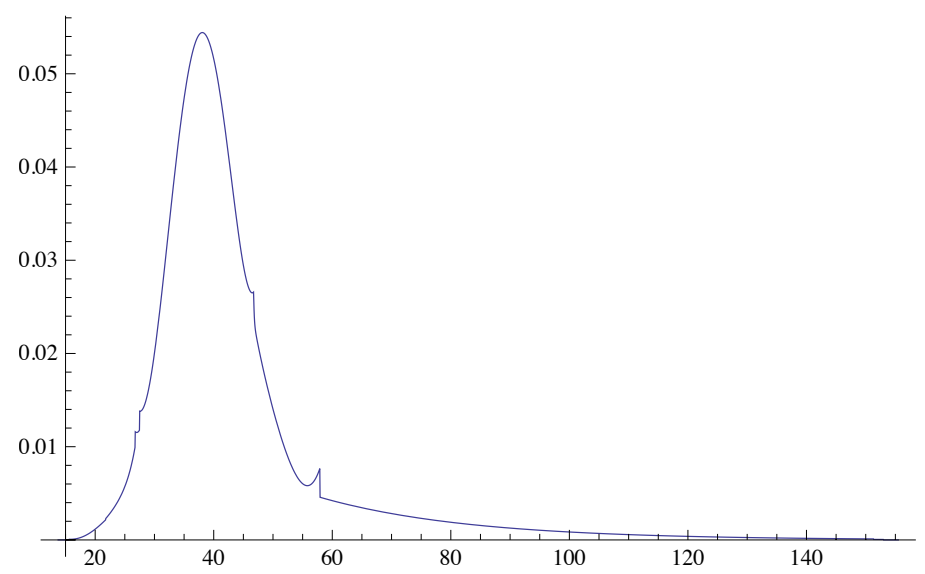

Figure 12: The MTE approximation of the marginal of $F$. 
for the mean of $\mathrm{F}$ was $(36.15,36.23)$, whilst for the standard deviation the result was $(20.25,20.31)$.

Notice that we are able to compute the solution using Monte Carlo simulation just because in this example no observed variables are considered. If some of the variables were observed, then the Monte Carlo approximation may not converge. As an illustration, consider a piece of a Bayesian network with three continuous variables $A, B$ and $C$, where $C=A+B$, i.e. $C$ has a deterministic conditional, and $A$ and $B$ take values on the interval $(0,1)$. Assume that $C$ is observed to be equal to the value $C=0.5$. In this scenario, it is likely that many of the configurations generated during the Monte Carlo sampling are finally discarded because they are incompatible with the observation. For instance, if we have sampled a value $A=0.7$ then any value sampled for $B$ will be incompatible with the observation $C=0.5$, as $A+B$ will be greater than 0.5 with total certainty.

Unlike Monte Carlo approximations, the solution we propose in this paper is not affected by evidence.

\section{Summary and Conclusions}

We have described some practical issues in solving hybrid BNs that include deterministic conditionals using MTEs and MOPs. As an illustration, we have solved a PERT hybrid BN consisting of 2 discrete and 10 continuous variables, 5 of which have linear deterministic conditionals.

One key observation is that in the process of solving the PERT hybrid $\mathrm{BN}$, some of the intermediate potentials have a large number of pieces, some of which are defined on lower dimensions and which have no useful information. One solution to this is to re-approximate these potentials with a smaller number of pieces and fewer degrees/terms. In the case of MOPs/MTEs, this can be done by dropping pieces on lower-dimensional regions (that have no probabilities) or pieces that have very small probabilities. In the case of MOPs, this can also be done using LIPs with Chebyshev points. In the case of MTEs, this can be done using the LM algorithm.

Some limitations of our methods are as follows. Currently, doing the re-approximations needs manual interventions to determine the number of pieces, the split points defining the pieces, and the number of terms in the case of MTEs. More work needs to be done in making these judgments.

We plan to solve the PERT hybrid BN using MOPs defined on hyperrhombuses to keep the number of pieces to a minimum, and compare the running time and accuracy with the corresponding results using hypercubes. Shenoy ${ }^{12}$ describes the use of LIPs with Chebyshev points for approximating univariate and bivariate functions by MOPs. However, more work needs to be done in re-approximating high-dimensional joint and conditional functions by MOP using LIPs with Chebyshev points. 


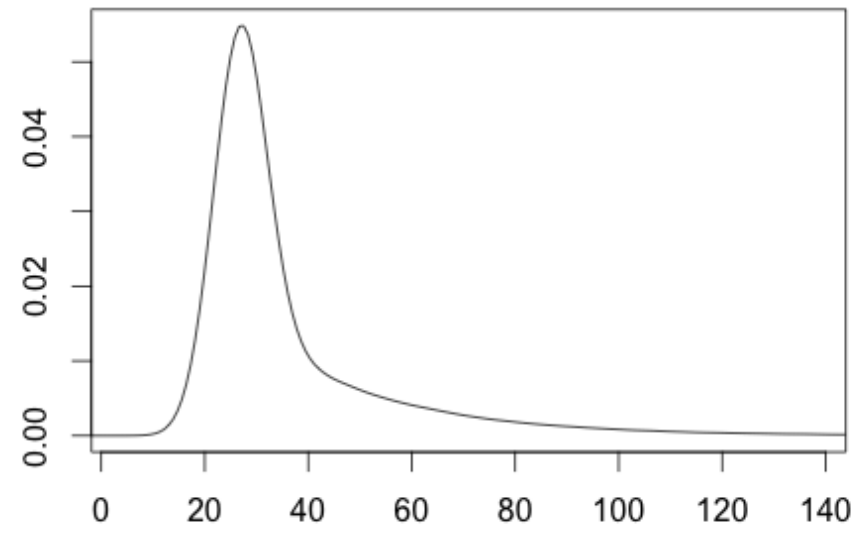

Figure 13: A Kernel approximation of the marginal PDF of $F$. 


\section{Acknowledgment}

This work has been supported by the Spanish Ministry of Economy and Competitiveness, through projects TIN2010-20900-C04-01 and TIN201020900-C04-02, by Junta de Andalucía through project P11-TIC- 7821 and by ERDF (FEDER) funds, and by a sabbatical partially funded by the University of Kansas, Lawrence, KS, to one of the co-authors. Thanks to Serafín Moral for suggesting the re-approximation strategy. Some portions of this paper have appeared in the proceedings of the ISDA'2011 conference and PGM'2012 workshop. ${ }^{28,29}$

\section{References}

1. Lauritzen S. Propagation of probabilities, means and variances in mixed graphical association models, J Am Statist Assoc 1992; 87:1098-1108.

2. Lerner U, Parr R. Inference in hybrid networks: Theoretical limits and practical algorithms. In: Breese J, Koller D, editors. Uncertainty in Artificial Intelligence: Proceedings of the Seventeenth Conference. Morgan Kaufmann, San Francisco, CA; 2001. pp. 310-318.

3. Moral S, Rumí R, Salmerón A. Mixtures of truncated exponentials in hybrid Bayesian networks. ECSQARU'01. Lect Notes Artif Intell 2143. 2001. pp. 135-143.

4. Shenoy PP, Shafer G. Axioms for probability and belief function propagation. In: Shachter R, Levitt T, Lemmer J, Kanal L., editors. Uncertainty in Artificial Intelligence 4. North Holland, Amsterdam; 1990. pp. 169-198.

5. Shenoy PP, West JC. Extended Shenoy-Shafer architecture for inference in hybrid Bayesian networks with deterministic conditionals. Int $\mathrm{J}$ Approx Reason 2011;52:805-818.

6. Zhang N, Poole D. Exploiting causal independence in Bayesian network inference. J Artif Intell Res 1996;5:301-328.

7. Cobb BR, Shenoy PP, Rumí R. Approximating probability density functions with mixtures of truncated exponentials. Stat Comput 2006;16:293-308.

8. Neil M, Tailor M, Marquez D. Inference in hybrid Bayesian networks using dynamic discretization. Stat Comput 2007;17:219-233.

9. Rumí R, Salmerón A. Approximate probability propagation with mixtures of truncated exponentials. Int J Approx Reason 2007;45:191-210. 
10. Langseth H, Nielsen TD, Rumí R, Salmerón A. Inference in hybrid Bayesian networks. Reliab Eng Syst Safe 2009;94:1499-1509.

11. Shenoy PP, West JC. Inference in hybrid Bayesian networks using mixtures of polynomials. Int J Approx Reason 2011;52:641-657.

12. Shenoy P. Two issues in using mixtures of polynomials for inference in hybrid Bayesian networks. Int J Approx Reason 2012;53:847-866.

13. Langseth H, Nielsen TD, Rumí R, Salmerón A. Mixtures of truncated basis functions. Int J Approx Reason 2012;53:212-227.

14. Marquez D, Neil M, Fenton N. Improved reliability modeling using Bayesian networks and dynamic discretization. Reliab Eng Syst Safe 2010;95:412-425.

15. Cinicioglu EN, Shenoy PP. Using mixtures of truncated exponentials for solving stochastic PERT networks. In: Kroupa T, Vejnarová J, editors. Proceedings of the 8th Workshop on Uncertainty Processing (WUPES09). University of Economics, Prague. 2009. pp. 269-283.

16. Cobb BR, Rumí R, Salmerón A. Modeling conditional distributions of continuous variables in Bayesian networks. Lect Comp Sci 2005;3646:3645 .

17. Langseth H, Nielsen TD, Rumí R, Salmerón A. Maximum likelihood learning of conditional MTE distributions. ECSQARU'09. Lect Notes Artif Intell 5590 2009; pp. 240-251.

18. Moral S, Rumí R, Salmerón A. Approximating conditional MTE distributions by means of mixed trees. ECSQARU'03. Lect Notes Artif Intell 2711 2003; pp. 173-183.

19. Xu Y. Lagrange interpolation on Chebyshev points of two variables. J Approx Theory 1996;87:220-238.

20. Levenberg K. A method for the solution of certain non-linear problems in least squares. Q Appl Math 1944;2:164-168.

21. Marquardt D. An algorithm for least-squares estimation of nonlinear parameters, SIAM J Appl Math 1963;11:431-441.

22. Langseth H, Nielsen TD, Rumí R, Salmerón A. Parameter estimation and model selection for mixtures of truncated exponentials. Int J Approx Reason 2010;51:485-498.

23. Rumí R, Salmerón A, Moral S. Estimating mixtures of truncated exponentials in hybrid Bayesian network. Test 2006;15:397-421. 
24. Langseth H, Nielsen TD, Pérez-Bernabé I, Salmerón A. Learning mixtures of truncated basis functions from data. Int $\mathrm{J}$ Approx Reason 2014;55:940-956.

25. Cho S. A linear Bayesian stochastic approximation to update project duration estimates. Eur J Oper Res 2009;196:585-593.

26. Malcolm DG, Roseboom JH, Clark CE, Fazar W. Application of a technique for research and development program evaluation. Oper Res 1959;7:646-669.

27. Khodakerami V, Fenton N, Neil M. Project scheduling: Improved approach to incorporating uncertainty using Bayesian networks. Proj Man J 2007;38:39-49.

28. Shenoy PP, Rumí R, Salmerón A. Some practical issues in inference in hybrid Bayesian networks with deterministic conditionals. In: Ventura S, Abraham A, Cios K, Romero C, Marcelloni F, Benitez JM, Gibaja E, editors. Proceedings of the 2011 Eleventh International Conference on Intelligent Systems Design and Applications. IEEE Research Publishing Services. Piscataway, NJ, 2011. pp. 605-610.

29. Rumí R, Salmerón A, Shenoy PP. Tractable inference in hybrid Bayesian networks with deterministic conditionals using re-approximations. In: Cano A, Gómez-Olmedo M, Nielsen TD, editors. Proceedings of the 6th European Workshop on Probabilistic Graphical Models. Granada, Spain, 2012. pp. 275-282. 